\title{
The Asymmetric Responses of Stock Markets
}

\author{
Abderrazak Dhaoui \\ Ipag Business School, University of Sousse, Tunisia \\ Stéphane Goutte \\ Université Paris 8 (LED), Paris, France \\ Khaled Guesmi \\ Ipag Business School, Paris, France
}

\begin{abstract}
This paper investigates how oil price shocks interact with oil-importing and oil-exporting stock markets within a nonlinear autoregressive distributed lag framework. By defining oil prices as endogenous variables, this model allows us to gage the shock transmission among the system variables and consider the asymmetric long- and short-run effects. Our empirical findings show an asymmetric long-run relation between stock market prices and macroeconomic fundamentals. These results suggest that investors should adjust their investment strategies to changes in oil prices and consider the asymmetry when forecasting and managing the negative impacts of unexpected events.
\end{abstract}

\section{JEL Classification: G1, E4, Q4}

Keywords: Oil Price Shocks, Stock Markets, Nonlinear Autoregressive Distributed Lag, Dynamic Multiplier

\footnotetext{
* Corresponding Author: Abderrazak Dhaoui; Ipag Business School (IPAG Lab), University of Sousse, Tunisia , Tel: 0033149407394, E-mail: abderrazak.dhaoui@yahoo.fr

Co-authors: Stéphane Goutte; Université Paris 8, 2 rue de la liberté, 93526 Saint-Denis, France and Paris School of Business, PSB, 59 rue Nationale, 75013 Paris, France.

Khaled Guesmi; IPAG Business School, 184 Bd Saint Germain 75006 Paris, E-mail: khaled.guesmi@ipag.fr
} 


\section{Introduction}

This study investigates the impact of oil price changes and certain macroeconomic variables on stock market prices. Investors are expected to react more when there are large negative oil price shocks. Thus, it might be inappropriate to gage such reactions in a linear setting. Further, this sample is marked by the global financial crisis of the autumn of 2008, which may have induced nonlinearity and asymmetry into the financial and economic time series. Various studies have found evidence of possible nonlinearity in financial and macroeconomic data (Aloui et al. 2013, Reboredo and Rivera-Castro 2013, Jammazi et al. 2014, among others). The possible nonlinearity is driven, according to Jammazi et al. (2014), "by successive episodes of economic and financial crisis, black swan events, geopolitical tensions, structural changes in business cycle, and heterogeneous economic agents." The authors also added, "the asymmetries can arise from the differences in the fundamental factors that determine the dynamics of markets under consideration". Accordingly, considering these vital externalities, we investigate the instantaneous long- and short-run asymmetric impacts of positive and negative unit changes in both national and world of both national and world oil price shocks on the stock market prices of oil-importing and oil-exporting countries, using the Nonlinear Autoregressive Distributed Lag (NARDL) bounds-testing approach proposed by Shin et al. (2014).

Using monthly data of Organization for Economic Cooperation and Development (OECD) oil-importing and oil-exporting countries from January 1986 to May 2015, the empirical findings show the existence of a long-run relation between stock market prices and oil prices, real industrial production and short-term interest rates. In particular, an asymmetric long-run relation is detected in most of the sampled countries. The dynamic multiplier also shows a significant and rapid response of stock market prices to positive and negative changes in the short-term interest rates. However, changes in oil price and real industrial production do not have a significant impact or a delayed time horizon impact on stock market prices. The study focuses on the OECD countries because they represent more than $50 \%$ of the world's oil consumption (53\% in 2010). ${ }^{1}$ They also have much lower oil consumption growth than non-OECD countries, demonstrating a

(Source) US Energy Information Administration (update: May 10, 2016). 
decline from 2000 to $2010 .^{2}$ These specific transitions in oil consumption are likely to induce asymmetric and nonlinear oil price impacts on stock market prices in the OECD countries.

From an economic perspective, a number of studies have focused on the linkages between changes in oil prices and economic recessions. In a seminal study, Hamilton (1983) linked the US economic recessions to rises in oil prices. He argued that seven of the eight US post-war economic recessions were preceded by an increase in oil prices (Hamilton 2011). Moreover, the negative impact of oil price shocks has been empirically established by Hamilton $(1983,1996,2003,2011)$ for the US, Cũnado and Perez de Gracia (2003) for European countries, Cũnado and Perez de Gracia (2005) for Asian economies, and Engemann et al. (2011) for other economies.

From a financial perspective, changes in crude oil prices may lead to economic depression, which could weaken asset prices. Thus, it is crucial to study the possible effects of crude oil price shocks on stock prices. These findings can help government authorities to reduce the instability in financial markets caused by oil price shock. Furthermore, an empirical analysis of the impact of oil price shocks on stock markets will help financial market participants in adjusting their decisions and revising their coverage of energy policies, which is substantially affected by the turbulence and uncertainty in the crude oil market (Arouri et al. 2011, Awartani and Maghyereh 2013).

Empirical evidence regarding the impact of oil price changes on stock markets is mixed and inconclusive. For example, Jones and Kaul (1996), Sadorsky (1999), and Cũnado and Perez de Gracia (2014) have confirmed that an increase in oil prices has a significant but negative impact on stock market prices. In contrast, several studies (Faff and Brailsford 1999, Sadorsky 2001, El-Sharif et al. 2005) have found a positive and significant relation between oil prices and stock market prices. An insignificant effect of oil prices on stock market prices has also been empirically confirmed by Chen et al. (1986) and Huang et al. (1996). Narayan and Sharma (2011) argued that oil prices may have different impacts on stock market prices, depending on the industries, and further reported that stock market prices are sensitive to lagged oil prices. Degiannakis et al. (2003), Kilian and Park (2009), Filis et al. (2011), Cũnado and Perez de Gracia (2014), and Dhaoui and Saidi (2015) noted that the effect of oil price shocks on stock prices depends on the nature of the shocks, namely, whether they are demand or supply side shocks. More obviously, the relation between oil price shocks and stock market prices

(Source) US Energy Information Administration (update: May 10, 2016). 
depends on whether a country is a net importer or net exporter of oil (Degiannakis et al. 2003, Filis et al. 2011, Dhaoui and Saidi 2015).

The remainder of this paper proceeds as follows. In Section II, we provide an overview of the related literature. Section III describes the data and methodology. In Section IV, we present the key empirical findings with a discussion, and finally, Section $\mathrm{V}$ concludes.

\section{Literature Review}

A negative relation between oil price changes and stock market prices is empirically, but strongly, confirmed in the US market, European countries, and other economies (Hamilton 1983, 1996, 2003, 2011, Jones and Kaul 1996, Sadorsky 1999, Cũnado and Perez de Gracia 2003, Cũnado and Perez de Gracia 2005, Engemann et al. 2011, Cũnado and Perez de Gracia 2014). A positive but significant impact of oil price changes on stock market prices has been reported by Faff and Brailsford (1999), Sadorsky (2001), and ElSharif et al. (2005), among others, but an insignificant relation between these variables has also been exposed by Chen et al. (1986) and Huang et al. (1996).

A number of transmission channels have also been identified, such as those by Bernanke et al. (1997), Lee and Ni (2002), Edelstein and Kilian (2007), Blanchard and Gali (2009), Kilian and Park (2009), Lee et al. (2011), and Serletis and Elder (2011). Stakeholders in oil markets are generally interested in how the volatility and oil price shocks are transmitted to stock market prices. Uncertainty is presented as an essential channel through which changes in oil prices can be transmitted to the key sectors of an economy, including the real sector and the financial sector (Başkaya et al. 2013, Aye 2015, Caporale et al. 2015, and Cũnado et al. 2015). In this vein, the stock market prices depend on the expected cash flows discounted by the required rate of returns (Williams 1938), which are substantially sensitive to any factor that could alter the expected cash flows or the required rate of returns (Filis et al. 2011). Moreover, a rise in oil prices can directly increase the cost of production and, consequently, lower the value of the cash flows that are considered in stock assessment models (Jones et al. 2004). These effects can also be extended to sectors other than the manufacturing industry. Indeed, due to a reduction in discretionary income or an increase in precautionary saving, an increase in oil prices may lead consumers to cut their spending that are not directly related to the 
oil industry (Gogineni 2010). However, oil price fluctuations can affect macroeconomic variables, including GDP growth, inflation, and the currency exchange rate (Hamilton and Herrara 2004, Hamilton 2005). Thus, oil price fluctuations lead to an increase in equity risk premiums, which can in turn affect the discount rates applied to cash flows in stock assessment models. With the same alignment, policy makers and central banks consider the increase in oil prices to be inflationary. Therefore, central banks react by increasing interest rates, particularly short-term interest rates, affecting the discount rate used in stock market price assessment models (Basher et al. 2012). Investors may also require an increase in the risk premium on the assets that they hold and experience greater exposure because of oil price fluctuations. Thus, an increase in the required risk premium on the volatility of oil prices leads to a significant response in equities. In this vein, French et al. (1987) found that the expected market risk premium and the predictable volatility of stock returns are positively related.

Faff and Brailsford (1999) and Jalil et al. (2009), have claimed that oil prices affect both consumers and producers. Faff and Brailsford (1999) documented that an increase in oil prices induces an increase in the prices of goods and services for consumers. In contrast, a decline in the demand for goods and services due to the inflationary effect driven by an increase in oil prices reduces the profits and lowers the magnitude of operations of producers. ${ }^{3}$ Jalil et al. (2009) argued that on the producer's side, " $a$ higher oil price is associated with higher input price." They added that an increase in production costs "will not only cause reduction in the quantity of output produced, but also push the price of output sold in the market to be higher." In fact, an increase in the cost of production and distribution due to a higher oil price will lead to a lower real income for producers. To protect their real income, producers will consequently pass on the cost to consumers. As a result, the general price level in an economy seems to increase in a similar manner.

Specifically, signs of nonlinearity have been reported and the responses are likely to be raised asymmetrically (Hamilton 2003, Lardic and Mignon 2006, 2008, Zhang 2008, Cologni and Manera 2009). Other types of interactions are empirically reported drivers, despite the importance of the studies, with mixed and inconclusive results obtained for the types of actions taken in response to the upheavals in oil prices. Different reasons exist for these mixed results. First, the samples covering periods and countries were

\footnotetext{
${ }^{3}$ The linkages between oil price increases and decreases and consumers and producers of goods and services were also discussed by Jalil et al. (2009).
} 
not equivalent. Second, the econometric analysis methods varied. Furthermore, the specification of oil price shocks faces several difficulties, and distinguishing between net oil-importing and net oil-exporting countries is not easy. It is a subject of confusion in that the needs and reserves of oil vary according to the country over time, as do the rate of consumption, the stability of producer countries, and the pressures of supply and global demand. Various empirical studies have found that stock market prices are asymmetrically affected by different exogenous regressors, such as financial news (Antoniou et al. 1998), stock market indices of foreign countries (Bahng and Shin 2003), and monetary policies (Tsai 2013). In particular, Tsai (2013) examined "whether a high oil price event that worsens the quality of a firm's balance sheet in turn provides an additional transmission channel to the stock market, which then affects stock returns." This author examined the asymmetric impacts of monetary shocks on stock returns across high oil price events and non-high oil price events over the period from 1995 to 2008. The "findings suggest that more energy-intensive industries and durable-goods industries react more significantly to monetary shocks based on high oil price events than on those based on non-high oil price events." Hence, oil prices, short-term interest rates, and real industrial production are possible candidates for causing asymmetric impacts on stock market prices.

Keeping the possible asymmetry in the reaction of stock markets, investigating stock markets' reactions to oil price shocks, short-term interest rates, and real industrial production can provide a better understanding of their relation. In particular, the NARDL approach adopted in this study allows the possible asymmetry in both long- and short-run effects to be considered. To the best of our knowledge, the existing empirical literature lacks evidence on the nonlinear relation between stock markets and oil price shocks. This study fill the gap by investigating the relation between oil price shocks and stock markets, considering nonlinearity and asymmetry for oil-importing and oilexporting countries. 


\section{Methods}

\section{A. Data description}

We use monthly data for real stock prices, real industrial production, nominal interest rates, and oil prices during the period from January, 1986, to May, 2015. The monthly data were used previously by Sadorsky (1999), Driesprong et al. (2008), Park and Ratti (2008), Lee et al. (2012), and Cũnado and Perez de Gracia (2014). The countries included in this study are Denmark, Mexico, Poland, and Portugal as net oil exporters and Estonia, France, Germany, Italy, Japan, South Korea, New Zealand, Austria, and the US as net oil importers. The data for oil prices and oil production are available from the Energy Information Administration (EIA) database. The data on industrial production, short-term interest rates, consumer price indices, and exchange rates are compiled from the OECD database and Global Financial Data (GFD). The data for stock market indices are available in the OECD and EUROSTAT databases. Furthermore, the empirical models include one endogenous variable, namely the real stock price $(s p)$, and numerous exogenous variables, specifically industrial production ( $i p$ ), the short-term interest rate $(r)$, and the national (world) oil price. Industrial production and short-term interest rates allow us to control for the indirect macroeconomic channels through which oil price changes are transmitted to stock markets. The oil price allows us to supervise the direct impact of oil price changes on stock market indices. Table 1 summarizes the selected variables and their formulas. 
Table 1. Selected variables and their formulas

\begin{tabular}{|c|c|c|c|}
\hline $\begin{array}{c}\text { Name of } \\
\text { variables }\end{array}$ & Notation & Formulas & Earlier used by \\
\hline Real stock returns & $R s p$ & $\begin{array}{c}R_{t}=\left(\ln \left(P_{t}\right)-\ln \left(P_{t-1}\right)\right) \times 100, \\
\text { where } \mathrm{P}_{t} \text { represents the real stock } \\
\text { market index at the time } t .\end{array}$ & $\begin{array}{c}\text { Park and Ratti (2008), } \\
\text { Cũnado and Perez De Gracia (2014) }\end{array}$ \\
\hline $\begin{array}{c}\text { Real national } \\
\text { oil prices }\end{array}$ & $O p$ & $\frac{\text { nominal oil price } \times \text { exchange rate }}{\text { consumer price index }}$ & $\begin{array}{c}\text { Cũnado and Perez de Gracia (2003, } \\
\text { 2005, 2014), Engemannet al. (2011) }\end{array}$ \\
\hline $\begin{array}{c}\text { Real industrial } \\
\text { production }\end{array}$ & $R i p$ & $\frac{\text { nominal indestrial production }}{\text { consumer price index }}$ & $\begin{array}{c}\text { Sadorsky (1999), } \\
\text { ParkandRatti (2008), }\end{array}$ \\
\hline $\begin{array}{c}\text { Cũnado and Perez de Gracia (2014) } \\
\text { short-term nominal } \\
\text { interest rate }\end{array}$ & $R$ & Percentage & Cũnado and Perez de Gracia (2014) \\
\hline
\end{tabular}

(Note) The UK Brent nominal price is used as proxy for nominal oil price. World real oil price is computed as nominal oil price deflated by the US producer price index.

\section{B. Estimation model}

In the literature, oil price-stock market linkages have been examined using various time series techniques, such as Autoregressive Conditionally Heteroscedastic (ARCH) and Generalized Autoregressive Conditionally Heteroskedastic (GARCH) model, cointegration and the Vector Error Correction Model (VECM), the Vector Autoregression (VAR) model, and the Markov switching model. The major disadvantages of these techniques include the presumptions of a symmetric relation between oil prices and stock market prices, and the linearity of the relation, and the time-varying independence of the relation. New research in this field has attached greater importance to the nonlinear and asymmetric relation between the variables. In particular, Shin et al. (2014) developed an extension to the well-known Autoregressive Distributed Lag (ARDL) approach initiated by Pesaran and Shin (1999) and Pesaran et al. (2001), namely the Nonlinear Autoregressive Distributed Lag (NARDL). This technique allows the investigation of the asymmetric linkages between the $s p$ (as an exogenous variable) and each of the rip, ir, and $o p$ (as endogenous variables). The asymmetry in the relation between the dependent variable and each of the independent variables refers to the asymmetry in the impact of 
negative and positive changes of $1 \%$ in the independent variable on the real stock price as the dependent variable in both signs and magnitude. Further, it allows both longand short-run asymmetries to be captured in the predictor. The asymmetric long-run specification of oil prices and stock market prices are specified as shown in Equation (1).

$$
r s p_{t}=\alpha_{0}+\alpha_{1} r i p_{t}^{+}+\alpha_{2} \operatorname{rip}_{t}^{-}+\alpha_{3} r_{t}^{+}{ }_{t}+\alpha_{4} r_{t}^{-}+\alpha_{5} o p_{t}^{+}+\alpha_{6} o p_{t}^{-}+\varepsilon_{t}
$$

where $r s p$ is real stock prices and rip and $r$ capture the industrial production and short-term interest rate channels through which oil price changes are transmitted to stock market prices, and $o p$ is the real oil price. $\alpha=\left(\alpha_{0}+\alpha_{1}+\cdots+\alpha_{6}\right)$ represents a vector of the long-run parameters to be estimated. For each independent variable $i v_{i}$, increases $\left(i v_{i t}^{+}\right)$and decreases $\left(i v_{i t}^{-}\right)$are specified as follows:

$$
i v_{i t}^{+}=\left\{\begin{array}{cc}
\Delta i v_{i t} & \text { if } \Delta i v_{i t}>0 \\
0 & \text { otherwise }
\end{array}\right.
$$

and

$$
i v_{i t}^{-}=\left\{\begin{array}{c}
\Delta i v_{i t} \text { if } \Delta i v_{i t}<0 \\
0 \quad \text { otherwise }
\end{array}\right.
$$

Based on Equation (1), $\alpha_{1}, \alpha_{3}$, and $\alpha_{5}$ capture the long-run link between stock markets and increases in rip, $r$, and op, respectively, and $\alpha_{2}, \alpha_{4}$, and $\alpha_{6}$ capture the long-run relation between stock market prices and decreases in rip, $r$, and $o p$, respectively. The former are assumed to be negative and the latter are expected to be positive. Because investors are more sensitive to increases in production costs than they are to decreases, the impacts of oil price increases on long-run changes in stock markets seem to be greater than those of the same magnitude of oil price decreases. Accordingly, $\alpha_{1}, \alpha_{3}$, and $\alpha_{5}$ seem to be greater than $\alpha_{2}, \alpha_{4}$, and $\alpha_{6}$, reflecting the asymmetric long-run relation between the stock market returns and these selected variables. Furthermore, the ARDL setting of Equation (1) can be framed as follows:

$$
\begin{aligned}
\Delta s p_{t}= & \alpha+\beta_{0} \mathrm{sp}_{\mathrm{t}-1}+\beta_{1} \operatorname{rip}_{\mathrm{t}-1}^{+}+\beta_{2} \mathrm{rip}_{\mathrm{t}-1}^{-}+\beta_{3} \mathrm{ir}_{\mathrm{t}-1}^{+}+\beta_{4} \mathrm{ir}_{\mathrm{t}-1}^{-}+\beta_{5} \mathrm{op}_{\mathrm{t}-1}^{+}+\beta_{6} \mathrm{op}_{\mathrm{t}-1}^{-} \\
& +\sum_{i=1}^{m} \lambda_{i} \Delta r s p_{t-i}+\sum_{i=1}^{n}\left(\gamma_{i}^{+} \operatorname{rip}_{t-i}^{+}+\gamma_{i}^{-} \operatorname{rip}_{t-i}^{-}\right)+\sum_{i=1}^{p}\left(\theta_{i}^{+} r_{t-i}^{+}+\theta_{i}^{-} r_{t-i}^{-}\right) \\
& +\sum_{i=1}^{q}\left(\delta_{i}^{+} o p_{t-i}^{+}+\delta_{i}^{-} o p_{t-i}^{-}\right)+u_{t}
\end{aligned}
$$


In Equation (4), all the variables are defined as in Equation (1), and $m, n, p, q, s$, and $h$ represent the lag orders. $\alpha_{1}=\beta_{1} / \beta_{0}, \alpha_{3}=\beta_{3} / \beta_{0}$, and $\alpha_{5}=\beta_{5} / \beta_{0}$ capture, respectively, the aforementioned long-run impacts of increases in rip, $r$, and op on stock markets. In the same way $\alpha_{2}=\beta_{2} / \beta_{0}, \alpha_{4}=\beta_{4} / \beta_{0}$, and $\alpha_{6}=\beta_{6} / \beta_{0}$ capture, respectively, the longrun impacts of the decreases in rip, $r$, and op on stock returns. $\sum_{i=1}^{n} \gamma_{i}^{+}, \sum_{i=1}^{p} \theta_{i}^{+}$, and $\sum$ capture, respectively, the short-run impacts on stock markets of the increases in the following variables: rip, $r$, and op. Similarly, $\sum_{i=1}^{n} \gamma_{i}^{-}, \sum_{i=1}^{p} \theta_{i}^{-}$and $\sum_{i=1}^{q} \delta_{i}^{-}$capture, respectively, the short-run impacts on stock market prices of the decreases in the following variables: rip, $r$, and $o p$. In addition to the asymmetric long-run relation captured in Equation (1), Equation (4) allows the asymmetric impacts of changes to be captured in the selected explanatory variables on stock markets. However, the nonlinear ARDL can be applied regardless of whether the variables are $I(0)$ or $I(1)$. The presence of an $I(2)$ variable can affect the estimate output significantly and "renders the computed F-statistics for testing cointegration invalid" (Ibrahim 2015). As a consequence, we conduct ADF, PP, and Kwiatkowski-Phillips-Schmidt-Shin (KPSS) unit root tests to establish the order of integration of the variables. In the second step, Equation (4) is estimated using the standard OLS method. We proceed following Katrakilidis and Trachanas (2012) and Ibrahim (2015) to determine the final specification of the NARDL model. The general-tospecific procedure involves running the basic model and trimming the insignificant lags after each estimation until significant results are obtained for all the regressors.

Once the objective at the second step has been achieved, the third step is to test for the presence of cointegration among the variables. The bounds-testing approach of Pesaran et al. (2001) and Shin et al. (2014) is used. This approach is to apply a Wald F-test to verify whether the null hypothesis $\beta_{0}=\beta_{1}=\beta_{2}=\beta_{3}=\beta_{4}=\beta_{5}=\beta_{6}=0$ can be rejected. Once a cointegration relation has been identified, the next step is to examine the long- and short-run asymmetries in the relation between the stock markets and the variable of interest. Furthermore, the asymmetric cumulative dynamic multiplier impacts of a $1 \%$ change in each regressor can be derived as

$$
m_{i n}^{+}=\sum_{i=0}^{n} \frac{\partial y_{t+i}}{\partial i v_{i t-1}^{+}} \quad(n=0,1,2, \ldots)
$$

At the same time, the asymmetric cumulative dynamic multiplier impacts of a $1 \%$ change in can be derived as 


$$
m_{\text {in }}^{-}=\sum_{i=0}^{n} \frac{\partial y_{t+i}}{\partial i v_{i t-1}^{-}} \quad(n=0,1,2, \ldots)
$$

For relations Equation (5) and Equation (6), we note that, as $n \rightarrow \infty, m_{n}^{+} \rightarrow \alpha_{n}^{+}$, and $m_{n}^{-} \rightarrow \alpha_{n}^{-}($Ibrahim 2015).

\section{Empirical Results}

Table 2 reports the summary statistics of the monthly data of real stock markets, real industrial production, short-term interest rates, and national oil prices. The world oil price corresponds to the US national oil price. The statistics include the sample's mean, minimum, maximum, standard deviation, kurtosis, skewness, Jarque-Bera (J-B), and J-B $p$-values. The Japanese real stock price appears to have the highest standard deviation (35.95\%), whereas that of South Korea shows the lowest standard deviation (3.31\%). All the sampled countries, with the exception of Austria and Italy, appear to have positive average returns. However, the excess returns of Austria, Estonia, Japan, South Korea, New Zealand, Portugal, and the US display negative unconditional skewness. This result implies that the stock markets of these countries have higher probabilities of closing with negative returns. The kurtosis of all the countries' stock markets exhibits leptokurtic distribution. This result indicates that extreme values of stock market prices have high chances of occurring. The $p$-value of the Jarque-Bera test statistics reveals that the null hypothesis of the normal distribution of stock market prices is not rejected for all the sampled countries.

It also shows that the national oil price appears to have a positive average value for all the selected countries. The highest price is observed in Italy (7.2827) and the lowest price in Austria (0.0257). In addition, all the national oil prices display negative unconditional skewness, which implies that the probability of a decrease in the national oil prices is higher than the probability of an increase. The excess kurtosis of the national oil prices of all the sampled countries shows leptokurtic distribution. This implies that there is a prevalence of extreme values for oil prices. The $p$-value of the Jarque-Bera test statistics reveals that the null hypothesis of normal distribution of oil prices is rejected for all the sampled countries, except Japan. 
For the short-term interest rate, the descriptive statistics show that the US has the lowest average short-term interest rate $(0.17 \%)$ and that Mexico has the highest average short-term interest rate $(4.02 \%)$. The lowest volatile short-term interest rate is shown in Japan and the US (standard deviation $=0.0005$ ). The short-term interest rate displays positive unconditional skewness, which implies a higher probability of an increase in the short-term interest rate than that of a decrease. At the same time, based on the kurtosis statistics, we show that the leptokurtic distribution implies the prevalence of extreme short-term interest rates. The $p$-value of the Jarque-Bera test statistics reveals that the null hypothesis of the normal distribution of short-term interest rates is rejected at the 5\% significance level in Austria, Denmark, Estonia, France, Germany, Italy, and Portugal and rejected at the 10\% significance level in the US. We cannot reject the null hypothesis, however, in Japan, South Korea, Mexico, and New Zealand.

For real industrial production, a higher standard deviation is observed in Japan (0.1322) and a lower level in Austria (0.0215). Negative unconditional skewness is observed in Estonia, Japan, South Korea, and the US. The real industrial production of Austria, Denmark, France, Germany, Italy, Mexico, New Zealand, Poland, and Portugal displays positive unconditional skewness. As for the other variables of the model, real industrial production appears to have leptokurtic distribution for all the sampled countries. The $p$-value of the Jarque-Bera test statistics reveals the rejection of the null hypothesis stating the normal distribution of real industrial production, in all the sampled countries except Germany. 


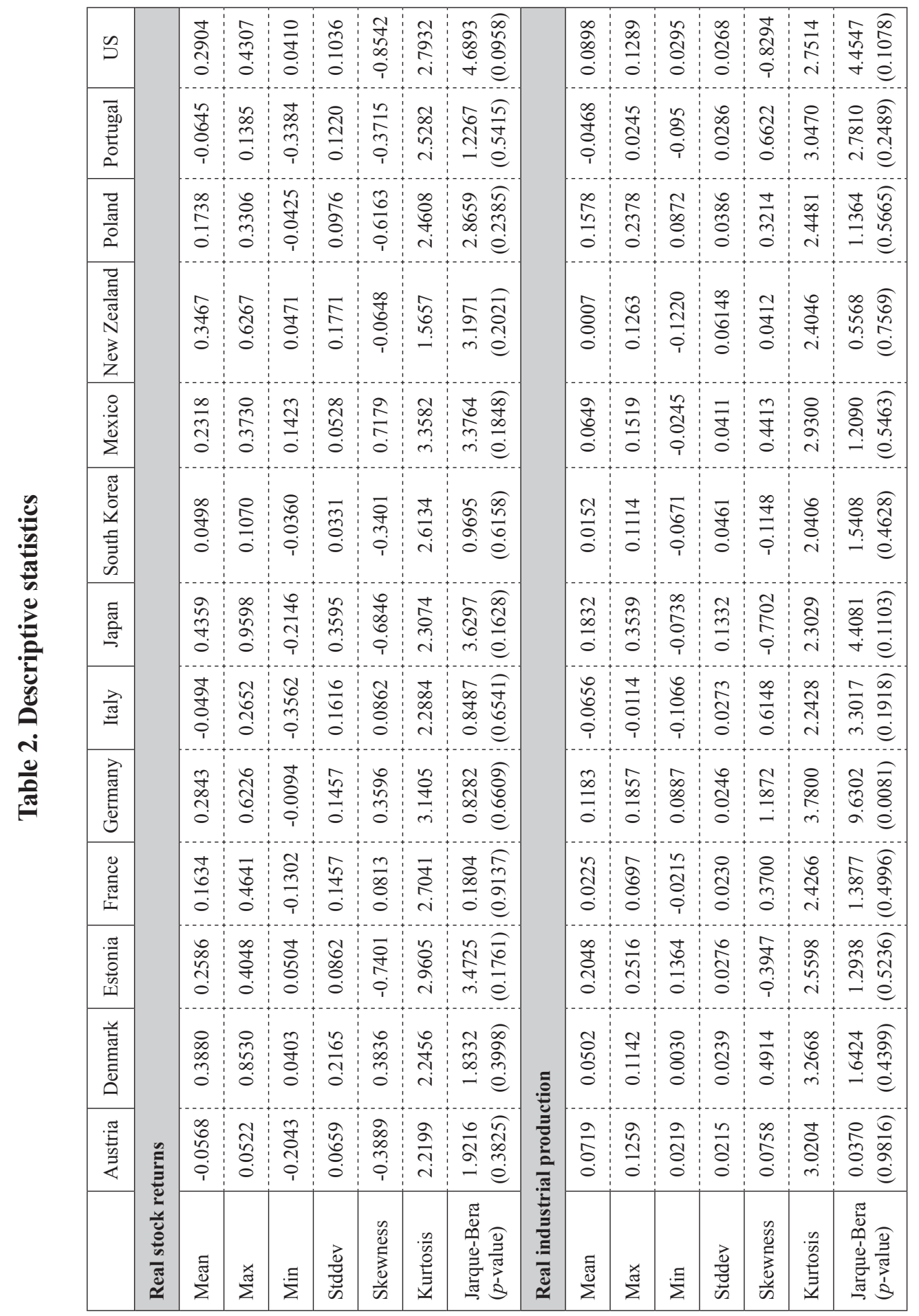




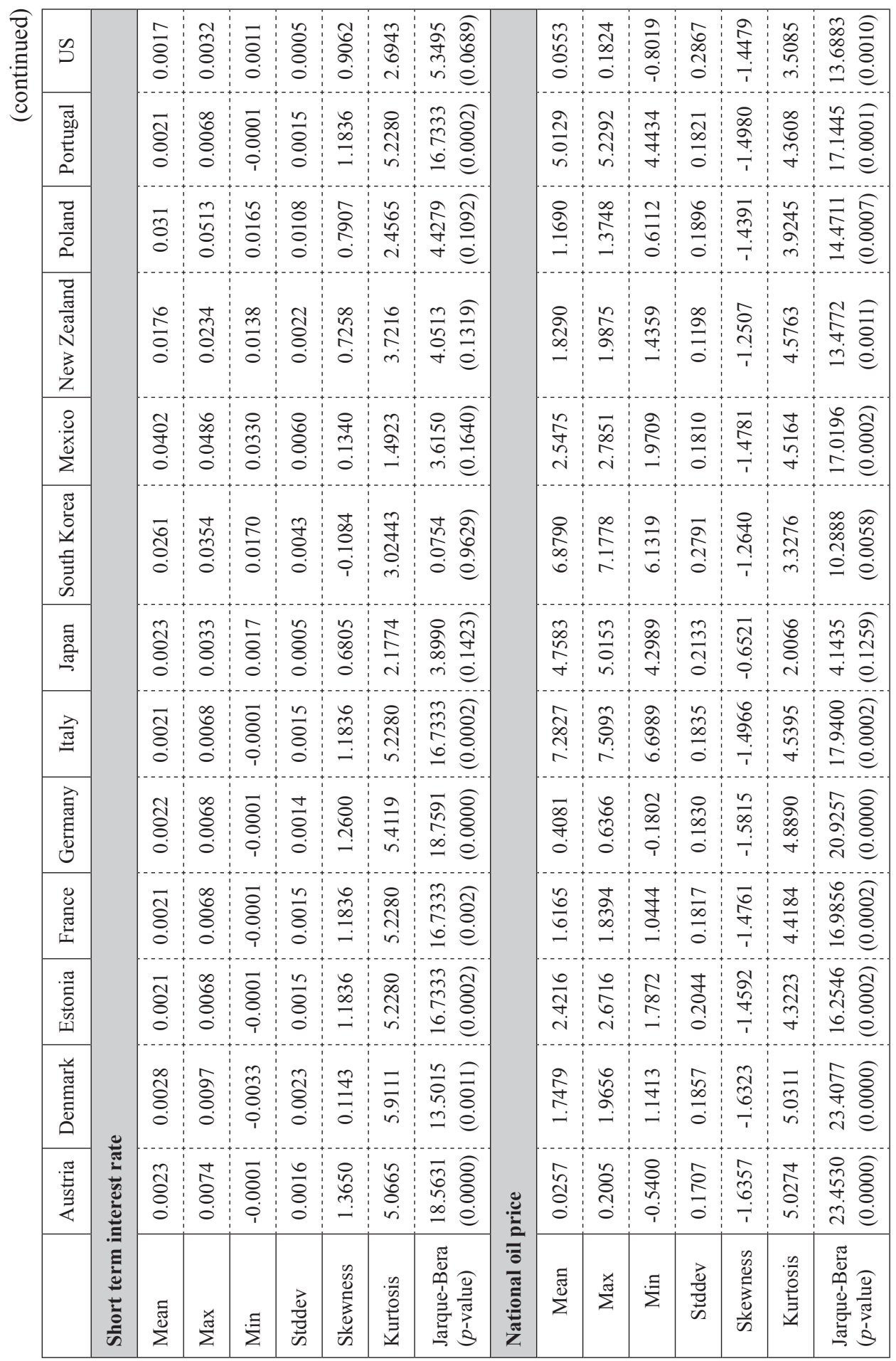


The bounds-testing approach requires that no I(2) variables are involved. Hence, we perform the unit root tests on the time series. We include both constant and trend terms and employ the Akaike Information Criterion (AIC) to select the optimal lag order in the ADF unit root test. For the selected OECD countries, the outcomes of the ADF, PhillipsPerron and KPSS unit root tests on the level of and for the first difference of the real stock market, short-term interest rate, real industrial production, and real oil prices are presented in Table 3. The various unit root tests indicate, for different countries, that the different series are integrated with an order of 1 , no series is $I(2)$. Hence, we can proceed to implement the bounds-testing approach. We estimate Equation (4) by applying the general-to-specific procedure to reach the final model specification.

The results of the cointegration bounds testing are shown in Table 4. We considered both national and world oil price specifications for each country. Based on the $F$-test results, the four variables, that is, stock market prices, oil prices, real industrial production, and short-term interest rates, move together in the long run. In fact, considering the national oil price specification, we show an $F$-statistic that is significant at the 5\% level for Austria, Estonia, Germany, Italy, Japan, South Korea, Mexico, New Zealand, Poland, and the US. For Denmark, France, and Portugal, the $F$-statistic is significant at the $10 \%$ level. For the world oil price specifications, the following results are shown: the $F$-test is significant at the 5\% level for Austria, Japan, South Korea, Poland, and the US. For Denmark, Estonia, France, Germany, Italy, Mexico, New Zealand, and Portugal, the $F$-test is significant at the $10 \%$ level. With these results, we can determine the effect of the stock market dynamics and their relation to positive and negative changes in oil prices on real industrial production and short-term interest rates. 


\begin{tabular}{|c|c|c|c|c|c|c|c|c|c|c|c|c|c|c|c|c|}
\hline & $\begin{array}{l}\tilde{\Omega} \\
\tilde{\Delta}\end{array}$ & & 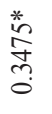 & 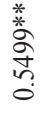 & $\begin{array}{l}\frac{*}{*} \\
\stackrel{*}{*} \\
\stackrel{0}{0} \\
0 \\
0\end{array}$ & 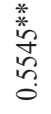 & \begin{tabular}{l}
$*$ \\
\multirow{2}{*}{} \\
$\delta$ \\
0 \\
0 \\
0
\end{tabular} & 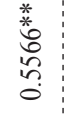 & $\begin{array}{l}\stackrel{0}{~} \\
\text { ปै } \\
\text { - }\end{array}$ & $\begin{array}{l}\vec{\sigma} \\
\stackrel{\sim}{0}\end{array}$ & 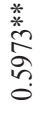 & 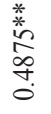 & 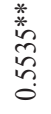 & $\begin{array}{l}\text { *. } \\
\stackrel{*}{\circ} \\
\infty \\
n \\
0 \\
0\end{array}$ & $\begin{array}{l}\stackrel{*}{*} \\
\stackrel{*}{\circ} \\
\stackrel{\infty}{0} \\
0\end{array}$ & 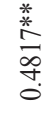 \\
\hline 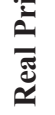 & $\hat{\mathrm{a}}$ & & 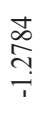 & $\underset{\sim}{\stackrel{n}{n}}$ & 옹 & 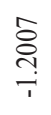 & 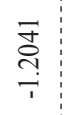 & $\stackrel{\mathscr{\infty}}{\stackrel{\infty}{+}}$ & $\begin{array}{l}\vec{D} \\
n \\
\rightarrow\end{array}$ & 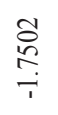 & $\begin{array}{l}\stackrel{1}{E} \\
\text { in } \\
i \\
i\end{array}$ & $\begin{array}{l}\stackrel{8}{\circ} \\
\stackrel{+}{+} \\
\rightarrow-1\end{array}$ & $\begin{array}{l} \pm \\
\infty \\
\infty \\
\infty \\
i\end{array}$ & 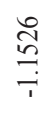 & \begin{tabular}{l}
0 \\
\multirow{2}{5}{} \\
$i$ \\
$i$ \\
$i$
\end{tabular} & 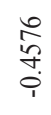 \\
\hline & 宸 & & 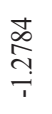 & 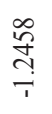 & 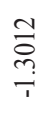 & $\begin{array}{l}\stackrel{a}{a} \\
\stackrel{0}{0} \\
\stackrel{0}{-}\end{array}$ & 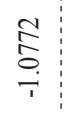 & 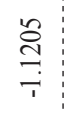 & 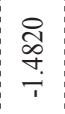 & $\underset{\substack{+ \\
\hdashline}}{\stackrel{\infty}{-}}$ & 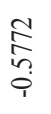 & 芯 & $\begin{array}{l}+ \\
\infty \\
\infty \\
\infty \\
i \\
i\end{array}$ & 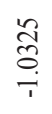 & 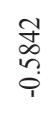 & 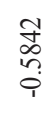 \\
\hline 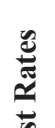 & $\begin{array}{l}\tilde{n} \\
\tilde{n} \\
\tilde{v}\end{array}$ & & \begin{tabular}{l}
$\frac{*}{*}$ \\
$\stackrel{*}{*}$ \\
\multirow{0}{*}{} \\
0 \\
0
\end{tabular} & \begin{tabular}{l}
$\frac{*}{*}$ \\
\multirow{2}{0}{} \\
$\tilde{n}$ \\
0
\end{tabular} & $\begin{array}{l}\text { *. } \\
\stackrel{*}{0} \\
\stackrel{\circ}{\circ} \\
0\end{array}$ & $\begin{array}{l}\text { *. } \\
\stackrel{*}{0} \\
0 \\
0 \\
0\end{array}$ & \begin{tabular}{l} 
* \\
\multirow{2}{*}{} \\
0 \\
0 \\
0
\end{tabular} & $\begin{array}{l}\text { * } \\
\stackrel{*}{0} \\
0 \\
0 \\
0\end{array}$ & \begin{tabular}{l}
$*$ \\
$\infty$ \\
\multirow{6}{*}{} \\
0
\end{tabular} & 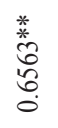 & $\begin{array}{l}\frac{*}{*} \\
\stackrel{*}{\circ} \\
\stackrel{8}{0} \\
0\end{array}$ & $\begin{array}{l}\stackrel{*}{*} \\
\stackrel{*}{\circ} \\
0\end{array}$ & $\begin{array}{l}\stackrel{*}{*} \\
\stackrel{*}{\hat{2}} \\
\stackrel{0}{0} \\
0\end{array}$ & $\begin{array}{l}\text { *. } \\
\text { 总 } \\
\stackrel{0}{0} \\
0\end{array}$ & $\begin{array}{l}\stackrel{*}{*} \\
\stackrel{*}{*} \\
\stackrel{*}{*}\end{array}$ & \\
\hline 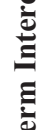 & $\hat{\mathrm{a}}$ & & $\begin{array}{l}\vec{F} \\
\overrightarrow{0} \\
\stackrel{p}{r}\end{array}$ & 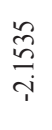 & 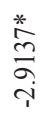 & 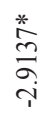 & 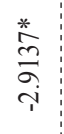 & 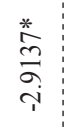 & $\begin{array}{l}\infty \\
n \\
\stackrel{n}{1}\end{array}$ & 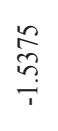 & $\begin{array}{l}\text { ڤ్ } \\
\text { I. } \\
\stackrel{1}{1}\end{array}$ & 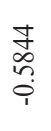 & 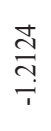 & $\begin{array}{l}\stackrel{*}{*} \\
\stackrel{0}{a} \\
\stackrel{\widehat{i}}{i}\end{array}$ & 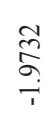 & \\
\hline कू & 宸 & & 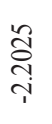 & $\begin{array}{c}\stackrel{n}{n} \\
\stackrel{n}{i}\end{array}$ & 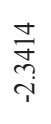 & $\underset{\substack{+i}}{\stackrel{\Delta}{\tau}}$ & $\begin{array}{l:}\vec{J} \\
\underset{T}{+}\end{array}$ & 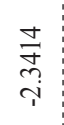 & 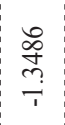 & $\underset{\substack{\infty \\
\rightarrow}}{\stackrel{\infty}{\vec{r}}}$ & 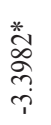 & $\begin{array}{l}\frac{a}{\mathbb{N}} \\
\stackrel{i}{i}\end{array}$ & $\frac{\infty}{n}$ & $\underset{i}{\stackrel{\Delta}{T}}$ & 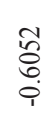 & \\
\hline 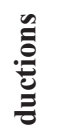 & $\begin{array}{l}\tilde{N} \\
\tilde{\Delta}\end{array}$ & בั๊ & 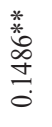 & $\frac{\text { 蒡 }}{\stackrel{+}{ \pm}}$ & $\begin{array}{l}\text { তू } \\
\text { o. } \\
0\end{array}$ & $\frac{\stackrel{a}{\Phi}}{0}$ & 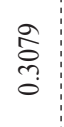 & 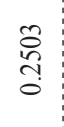 & 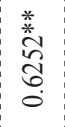 & $\begin{array}{l}\stackrel{*}{*} \\
\stackrel{0}{0} \\
\stackrel{0}{n} \\
0\end{array}$ & 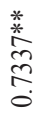 & $\frac{\stackrel{*}{\approx}}{\stackrel{\sigma}{\sigma}}$ & 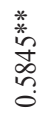 & $\begin{array}{l}\text { *. } \\
\text { Oें } \\
\text { ర్ర } \\
0\end{array}$ & $\frac{n}{\frac{n}{0}}$ & \\
\hline 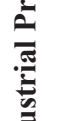 & $\hat{\mathbf{a}}$ & & $\begin{array}{l}\stackrel{n}{n} \\
\stackrel{n}{n} \\
i\end{array}$ & 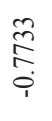 & 命 & $\begin{array}{l}\overrightarrow{0} \\
\overrightarrow{6} \\
\stackrel{+}{T}\end{array}$ & $\begin{array}{l}\hat{\alpha} \\
\text { ळे } \\
\text { oे }\end{array}$ & 高 & $\begin{array}{l}2 \\
o \\
0 \\
0\end{array}$ & 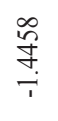 & 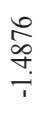 & $\begin{array}{l}\infty \\
\stackrel{2}{\alpha} \\
\stackrel{1}{i}\end{array}$ & $\begin{array}{l}\infty \\
\stackrel{6}{0} \\
\stackrel{n}{n} \\
i\end{array}$ & $\begin{array}{l}\text { *. } \\
\stackrel{\alpha}{\alpha} \\
\infty \\
i \\
i\end{array}$ & 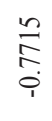 & \\
\hline 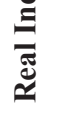 & 宸 & & $\begin{array}{l}8 \\
\frac{8}{0} \\
\frac{1}{1}\end{array}$ & 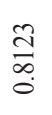 & $\frac{\vec{n}}{\stackrel{7}{c}}$ & $\begin{array}{l}\underset{*}{*} \\
\stackrel{0}{\infty} \\
\infty \\
i \\
i\end{array}$ & $\begin{array}{l}t \\
0 \\
0 \\
\end{array}$ & $\begin{array}{l}2 \\
\dot{j} \\
\stackrel{5}{7}\end{array}$ & \begin{tabular}{l}
$\stackrel{Y}{ \pm}$ \\
\hdashline
\end{tabular} & $\underset{\substack{n \\
i}}{\stackrel{N}{n}}$ & 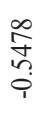 & $\begin{array}{l}\infty \\
\stackrel{0}{\infty} \\
\stackrel{2}{0} \\
i\end{array}$ & 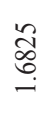 & $\begin{array}{l}\text { ते } \\
\text { ळ. } \\
-\end{array}$ & 孞 & \\
\hline & $\begin{array}{l}\tilde{n} \\
\tilde{n} \\
\tilde{\Sigma}\end{array}$ & & 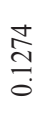 & 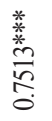 & 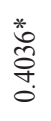 & $\begin{array}{l}\stackrel{*}{*} \\
\stackrel{2}{2} \\
\stackrel{2}{0}\end{array}$ & 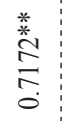 & $\begin{array}{l}\frac{*}{*} \\
\stackrel{5}{E} \\
\stackrel{0}{0}\end{array}$ & $\begin{array}{l}\frac{*}{*} \\
\frac{2}{2} \\
0 \\
0\end{array}$ & $\begin{array}{l}\frac{*}{*} \\
\stackrel{*}{0} \\
\stackrel{0}{0} \\
\stackrel{0}{0}\end{array}$ & $\begin{array}{l}\stackrel{*}{*} \\
\stackrel{*}{3} \\
\stackrel{0}{0}\end{array}$ & $\begin{array}{l}\stackrel{*}{\infty} \\
\stackrel{\infty}{*} \\
\stackrel{0}{0}\end{array}$ & $\frac{\frac{*}{*}}{\stackrel{0}{0}}$ & 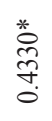 & 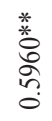 & \\
\hline 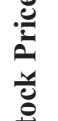 & $\hat{2}$ & & 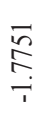 & $\begin{array}{l}n \\
\stackrel{2}{0} \\
\stackrel{2}{2}\end{array}$ & $\begin{array}{l}\stackrel{m}{ٍ} \\
\stackrel{\leftrightarrow}{i}\end{array}$ & 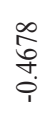 & $\begin{array}{l}2 \\
2 \\
0 \\
0 \\
1\end{array}$ & $\begin{array}{l}0 \\
\tilde{n} \\
\tilde{n} \\
i\end{array}$ & $\begin{array}{l}0 \\
0 \\
0 \\
0 \\
+ \\
1\end{array}$ & \begin{tabular}{l}
$\stackrel{\circ}{\circ}$ \\
\hdashline
\end{tabular} & 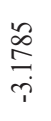 & $\begin{array}{l}\stackrel{0}{\circ} \\
\stackrel{n}{n} \\
\stackrel{1}{1}\end{array}$ & 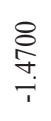 & $\underset{i}{\stackrel{P}{F}}$ & 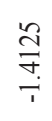 & \\
\hline & 宸 & & $\underset{\stackrel{a}{a}}{\stackrel{\vec{a}}{-1}}$ & 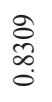 & 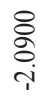 & 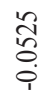 & $\begin{array}{l}\frac{8}{9} \\
0 \\
\end{array}$ & $\begin{array}{l}\text { ते } \\
\text { ลे } \\
\overline{1}\end{array}$ & 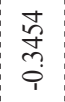 & $\begin{array}{l}\text { 옹 } \\
\text { तે }\end{array}$ & $\begin{array}{l}\stackrel{2}{\ni} \\
\underset{i}{i}\end{array}$ & $\begin{array}{c}\stackrel{n}{\hat{\infty}} \\
\infty \\
\stackrel{1}{1}\end{array}$ & $\begin{array}{l}\mathscr{8} \\
\stackrel{+}{+} \\
\stackrel{-}{1}\end{array}$ & $\begin{array}{l}\hat{\sigma} \\
\text { ô } \\
\text { i }\end{array}$ & $\underset{\mathfrak{\sigma}}{\stackrel{5}{\tau}}$ & \\
\hline & & & 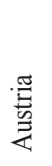 & $\begin{array}{l}\text { 节 } \\
\text { ดे }\end{array}$ & 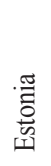 & 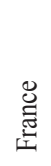 & 言 & 巴્ & 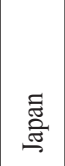 & 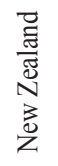 & 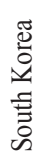 & 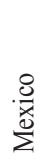 & $\begin{array}{l}\frac{\text { I }}{0} \\
\text { D. }\end{array}$ & 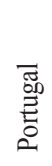 & $\Omega$ & 룰 \\
\hline
\end{tabular}




\begin{tabular}{|c|c|c|c|c|c|c|c|c|c|c|c|c|c|c|c|c|c|}
\hline \multirow{3}{*}{ 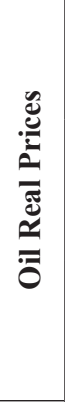 } & $\begin{array}{l}\tilde{n} \\
\hat{\tilde{a}}\end{array}$ & & 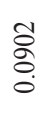 & $\begin{array}{l}\text { ర్ర } \\
\stackrel{0}{0} \\
0\end{array}$ & $\begin{array}{l}\stackrel{\infty}{\mathbb{N}} \\
\stackrel{0}{0} \\
0\end{array}$ & $\begin{array}{l}\hat{n} \\
\hat{0} \\
0\end{array}$ & $\begin{array}{l}\text { Dे } \\
\text { : } \\
0\end{array}$ & $\begin{array}{l}\stackrel{0}{0} \\
\stackrel{0}{0}\end{array}$ & $\begin{array}{l}\stackrel{2}{a} \\
\stackrel{a}{0}\end{array}$ & $\begin{array}{l}\overleftarrow{J} \\
\text { oे } \\
0\end{array}$ & $\begin{array}{l}+ \\
\stackrel{+}{\circ} \\
\stackrel{0}{0}\end{array}$ & $\begin{array}{l}0 \\
\text { ర్ర } \\
0 \\
0\end{array}$ & $\frac{\text { I }}{0}$ & $\begin{array}{l}\text { ֻे } \\
\infty \\
0 \\
0\end{array}$ & $\frac{\text { से }}{\frac{2}{0}}$ & $\frac{\stackrel{9}{0}}{0}$ & 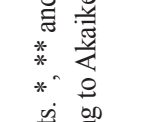 \\
\hline & $\hat{a}$ & & 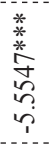 & 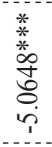 & $\begin{array}{l}\frac{*}{*} \\
\stackrel{*}{*} 0 \\
\stackrel{0}{\circ} \\
\stackrel{0}{+} \\
\dot{+}\end{array}$ & 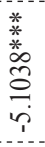 & 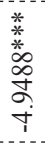 & 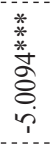 & 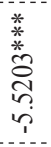 & 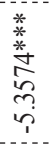 & 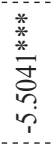 & 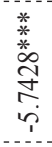 & 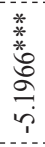 & 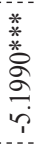 & 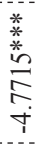 & 旁 & 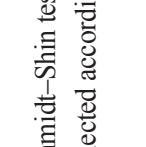 \\
\hline & 崇 & & 美 & 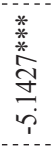 & $\begin{array}{l}\stackrel{*}{*} \\
\stackrel{*}{*} \\
\stackrel{+}{\sigma} \\
\stackrel{+}{\sigma}\end{array}$ & 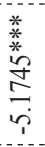 & \begin{tabular}{c}
$*$ \\
$*$ \\
\multirow{2}{*}{} \\
\multirow{2}{*}{} \\
$\dot{0}$ \\
$i$
\end{tabular} & 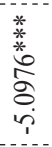 & \begin{tabular}{l} 
*ै \\
$\stackrel{*}{*}$ \\
\multirow{2}{*}{} \\
$i$ \\
$i$
\end{tabular} & 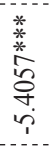 & 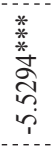 & 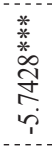 & 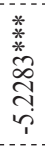 & 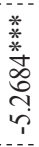 & 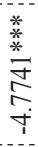 & 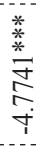 & 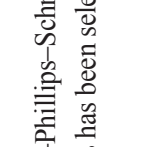 \\
\hline 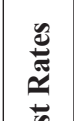 & $\begin{array}{l}\tilde{n} \\
\hat{\tilde{v}}\end{array}$ & & $\begin{array}{l}\overline{\widehat{o}} \\
\text { ơ }\end{array}$ & $\begin{array}{l}\stackrel{2}{\infty} \\
\stackrel{2}{0} \\
0\end{array}$ & $\frac{\widetilde{\Xi}}{\tilde{\sigma}}$ & 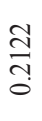 & 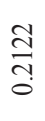 & $\frac{\widetilde{\Xi}}{\tilde{c}}$ & $\underset{\stackrel{5}{0}}{0}$ & $\stackrel{\infty}{\stackrel{\infty}{\infty}}$ & $\begin{array}{l}\frac{n}{5} \\
\stackrel{5}{0}\end{array}$ & $\stackrel{\infty}{\rightrightarrows}$ & $\begin{array}{l}\frac{n}{0} \\
0\end{array}$ & 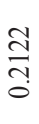 & 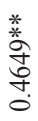 & & 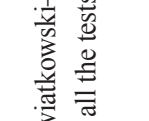 \\
\hline 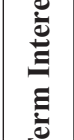 & $\hat{\mathrm{a}}$ & & 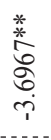 & 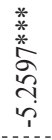 & 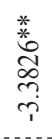 & \begin{tabular}{l}
$*$ \\
\multirow{*}{*}{} \\
$\infty$ \\
$\infty$ \\
$m$ \\
$?$ \\
1
\end{tabular} & 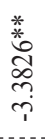 & 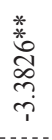 & 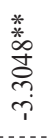 & 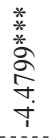 & 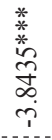 & $\begin{array}{l}\stackrel{*}{*} \\
\stackrel{*}{*} \\
\stackrel{*}{\sigma} \\
\dot{0} \\
\dot{y}\end{array}$ & 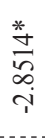 & 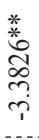 & 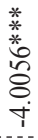 & & $\begin{array}{l}0 \\
0 \\
0\end{array}$ \\
\hline 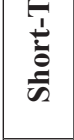 & 晏 & & $\begin{array}{l}\frac{*}{*} \\
* \\
\omega \\
\infty \\
\infty \\
\infty \\
\dot{1}\end{array}$ & \begin{tabular}{l}
$\frac{*}{*}$ \\
\multirow{2}{*}{} \\
2 \\
6 \\
0 \\
$i$ \\
$i$
\end{tabular} & $\begin{array}{l}\stackrel{*}{*} \\
\stackrel{*}{2} \\
\vec{\nabla} \\
\dot{1}\end{array}$ & 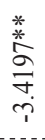 & 苦 & 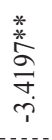 & $\begin{array}{l}\stackrel{*}{*} \\
\stackrel{*}{n} \\
\stackrel{n}{i}\end{array}$ & \begin{tabular}{l}
$\frac{*}{*}$ \\
$\stackrel{*}{*}$ \\
\multirow{f}{*}{} \\
$\stackrel{+}{+}$ \\
$\dot{+}$
\end{tabular} & 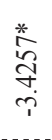 & 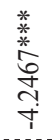 & 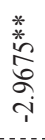 & 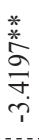 & $\begin{array}{l}* \\
\stackrel{*}{\circ} \\
\stackrel{0}{6} \\
i\end{array}$ & & $\frac{10}{b}$ \\
\hline .气. & $\begin{array}{l}\tilde{n} \\
\hat{\Sigma}\end{array}$ & 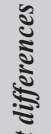 & 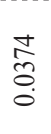 & 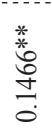 & $\begin{array}{l}\text { *. } \\
\text { 今े } \\
0 \\
0 \\
0\end{array}$ & 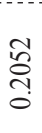 & 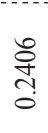 & 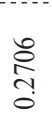 & $\frac{1}{0}$ & $\begin{array}{l}\overrightarrow{0} \\
\stackrel{0}{0}\end{array}$ & 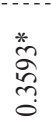 & ठृ. & $\begin{array}{l}\stackrel{\varpi}{6} \\
\stackrel{0}{0}\end{array}$ & $\begin{array}{l}\stackrel{8}{\circ} \\
\vdots \\
0\end{array}$ & 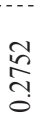 & & $\vec{\Xi}$ \\
\hline 离 & ฉి & $\stackrel{\Sigma}{*}$ & 美 & 苇 & 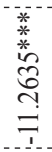 & 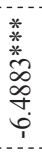 & 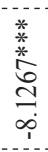 & 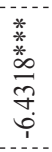 & 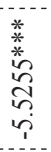 & $\frac{\frac{*}{*}}{\frac{*}{2}}$ & 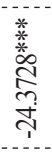 & 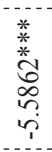 & $\begin{array}{l}\frac{*}{*} \\
* \\
* \\
\infty \\
\infty \\
\infty \\
\vdots \\
1\end{array}$ & 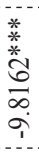 & 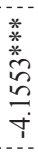 & & 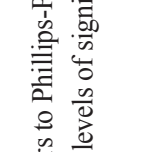 \\
\hline $\begin{array}{l}\stackrel{\Xi}{\Xi} \\
\overline{\mathscr{E}} \\
\simeq\end{array}$ & 岁 & & 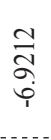 & 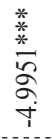 & 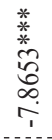 & 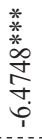 & $\begin{array}{l}\frac{*}{*} \\
* \\
8 \\
0 \\
0 \\
0 \\
1\end{array}$ & 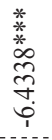 & 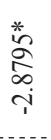 & $\frac{*}{*}$ & 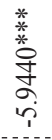 & $\begin{array}{l}\frac{*}{*} \\
\stackrel{*}{*} \\
\hat{0} \\
0 \\
\vdots \\
i\end{array}$ & $\stackrel{*}{\stackrel{*}{\sigma}}$ & $\begin{array}{l}* \\
* \\
\infty \\
\infty \\
\infty \\
= \\
i\end{array}$ & 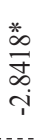 & & $\begin{array}{l}\overrightarrow{0} \\
\text { so } \\
\text { so in }\end{array}$ \\
\hline & $\begin{array}{l}\tilde{v} \\
\tilde{\varepsilon} \\
\end{array}$ & & 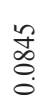 & $\begin{array}{l}\text { d } \\
\text { तิ }\end{array}$ & 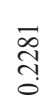 & $\begin{array}{l}\text { I } \\
\stackrel{8}{\circ} \\
0\end{array}$ & 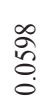 & $\begin{array}{l}\stackrel{+}{0} \\
\stackrel{0}{0} \\
0 \\
0\end{array}$ & $\begin{array}{l}\frac{J}{0} \\
0 \\
0\end{array}$ & $\begin{array}{l}0 \\
6 \\
0 \\
0\end{array}$ & 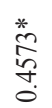 & 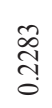 & $\stackrel{\frac{1}{m}}{\stackrel{m}{0}}$ & $\begin{array}{l}\hat{\delta} \\
\vdots \\
0\end{array}$ & స̃ & & $\stackrel{\stackrel{2}{.}}{.}$ \\
\hline 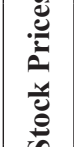 & 完 & & 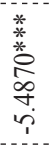 & 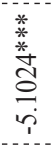 & $\begin{array}{l}* \\
* \\
* \\
* \\
0 \\
0 \\
0 \\
+ \\
+\end{array}$ & $\begin{array}{l}\frac{*}{*} \\
\frac{*}{*} \\
0 \\
0 \\
0\end{array}$ & 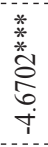 & 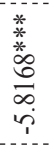 & 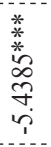 & 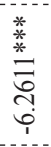 & 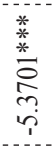 & 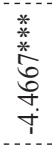 & 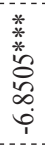 & 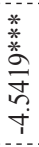 & 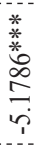 & & 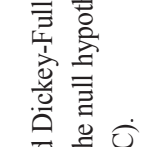 \\
\hline & 岁 & & 访 & 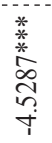 & 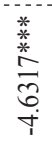 & $\frac{\stackrel{*}{*}}{\stackrel{*}{*}}$ & 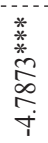 & 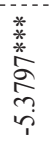 & 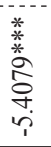 & 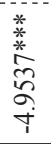 & 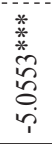 & 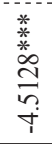 & 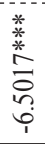 & 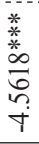 & 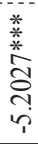 & & 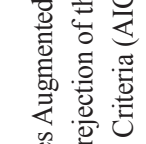 \\
\hline & & & 营 & 离 & 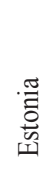 & 它 & 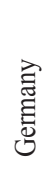 & 馬 & 胥 & 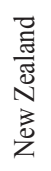 & 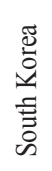 & 总 & $\approx$ & $\stackrel{\text { D. }}{2}$ & 2 & 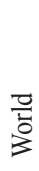 & 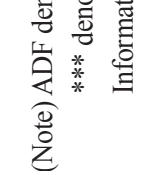 \\
\hline
\end{tabular}


Table 4. Nonlinear ARDL bounds test results

\begin{tabular}{|l|c|c|c|c|}
\hline & \multicolumn{2}{|c|}{ National oil price specification } & \multicolumn{2}{c|}{ World oil price specification } \\
\hline Countries & $F$-statistics & Cointegration & $F$-statistics & Cointegration \\
\hline Austria & $6.0629^{* * *}$ & Yes & $4.71^{* *}$ & Yes \\
\hline Denmark & $4.251^{*}$ & Yes & $4.149^{*}$ & Yes \\
\hline Estonia & $6.955^{* * *}$ & Yes & $4.307 *$ & Yes \\
\hline France & $3.903^{*}$ & Yes & $3.787^{*}$ & Yes \\
\hline Germany & $6.493^{* * *}$ & Yes & $4.037^{*}$ & Yes \\
\hline Italy & $4.488^{* *}$ & Yes & $3.356^{*}$ & Yes \\
\hline Japan & $5.996^{* * *}$ & Yes & $7.741^{* * *}$ & Yes \\
\hline South Korea & $6.418^{* * *}$ & Yes & $6.396^{* * *}$ & Yes \\
\hline Mexico & $5.231^{* *}$ & Yes & $4.244^{*}$ & Yes \\
\hline New Zealand & $4.521^{* *}$ & Yes & $3.819^{*}$ & Yes \\
\hline Poland & $6.232^{* * *}$ & Yes & $5.774^{* * *}$ & Yes \\
\hline Portugal & $3.278^{*}$ & No & $3.508^{*}$ & Yes \\
\hline United States & $6.939^{* * *}$ & Yes & $6.939^{* * *}$ & Yes \\
\hline
\end{tabular}

(Note) ${ }^{* * *}, * *$, and $*$, denote significance level at $1 \%, 5 \%$ and $10 \%$.

Before investigating the long- and short-run connections between the stock market prices and the variables of interest, in the first step, we judge the adequacy of the dynamic specification based on various diagnostic statistics, including the LM statistics for the order 12 (denoted SC (12)), the White test of heteroscedasticity and the Ramsey Regression Equation Specification Error Test (RESET) for the stability test. The results of these tests are presented at the bottom of Table 6 . The model with the national oil price specification passes all the diagnostic tests, suggesting the absence of autocorrelation at the $5 \%$ significance level for all the countries. At the same time, the model with the world oil price specification passes all the diagnostic tests, suggesting the absence of autocorrelation at the 5\% and 1\% significance levels for all countries except Poland. For the stability test diagnostic, the results of the RESET test suggest that we cannot reject the null hypothesis that the model has no omitted variables at the $1 \%$ significance level, with both national and world oil price specifications, for the following economies: Denmark, France, Italy, Japan, South Korea, Mexico, Poland, and the US. For Estonia, Germany, Mexico, and New Zealand, however, we reject the null hypothesis in favor of 
the alternative, suggesting that the model has possible missing variables at the $1 \%$ level. At the 5\% significance level, the null hypothesis is also rejected for Mexico and Poland with the national oil price specification and for the US with both national and world oil price specifications. The results for Durbin-Watson statistics show the absence of autocorrelation for all the selected countries, with the exception of Japan having world oil price specification.

Table 5 summarizes the results of the Wald test of long- and short-run asymmetry. The analysis of the null hypothesis of symmetry in both the long and short run provides mixed results. The null hypothesis of symmetry in the long run against the alternative of asymmetry is tested using the Wald statistic, including $\mathrm{H}_{\mathrm{LR} \text {,rip }}$ : $\alpha_{1}=\alpha_{2}$ (i.e., $\left.\beta_{1} / \beta_{0}=\beta_{2} / \beta_{0}\right), \mathrm{H}_{\mathrm{LR}, \mathrm{r}}: \alpha_{3}=\alpha_{4}$ (i.e., $\beta_{3} / \beta_{0}=\beta_{4} / \beta_{0}$ ), and $\mathrm{H}_{\mathrm{LR}, \mathrm{op}}$ : $\alpha_{5}=\alpha_{6}$ (i.e., $\beta_{5} / \beta_{0}=\beta_{6} / \beta_{0}$ ), respectively. The results (Table 5 , long-run asymmetry) suggest the rejection of the null hypothesis for real industrial production in Austria, Estonia, Germany, the US, and Poland. The rejection of the null hypothesis of the symmetric long-run impact of positive and negative changes in the short-term interest rate is shown for Austria, Japan, New Zealand, the US, South Korea, and Poland. For negative and positive oil price shocks, the alternative hypothesis is confirmed for Austria, Estonia, Japan, the US, and Poland. However, we fail to reject the null hypothesis of a long-run symmetric impact of positive and negative changes in the different independent variables in the cases of France, Italy, Denmark, Mexico, and Portugal.

Regarding asymmetry in the short run, we analyze the null hypothesis of symmetry against the alternative of asymmetry based on the Wald statistic, including $\mathrm{H}_{\mathrm{SR} \text {.rip }}$ : $\sum_{i=1}^{n} \gamma_{i}^{+}=\sum_{i=1}^{n} \gamma_{i}^{-}, \mathrm{H}_{\mathrm{SR} . \mathrm{r}}: \sum_{i=1}^{n} \theta_{i}^{+}=\sum_{i=1}^{n} \theta_{i}^{-}$, and $\mathrm{H}_{\mathrm{SR} . \mathrm{op}}: \sum_{i=1}^{n} \delta_{i}^{+}=\sum_{i=1}^{n} \theta_{i}^{-}$. The results show the rejection of the null hypothesis of short-run symmetry in the positive and negative changes for real industrial production in Austria, Estonia, Germany, the US and Poland. For the short-term interest rate, the null hypothesis of symmetry is rejected for Austria, France, Japan, New Zealand, South Korea, the US, and Poland. We reject the null hypothesis of short-run symmetry in the positive and negative oil price shocks in Austria, Estonia, Italy, Japan, the US, Denmark, and Poland. Overall, the results show the asymmetric impact of positive and negative changes in at least one of the three independent variables of real industrial production, short-term interest rates, and oil prices in the sampled oil-importing countries. In oil-exporting countries, the main result confirms the presence of a symmetric impact, with the following exceptions: Poland, for which we reject the null hypothesis of symmetry for all three variables, and Denmark and Mexico, for the oil price and the short-term interest rate, respectively. 
Table 5. Long-run and short-run asymmetry tests

\section{A. National prices}

\begin{tabular}{|c|c|c|c|c|c|c|}
\hline \multirow[b]{2}{*}{ Countries } & \multicolumn{3}{|c|}{ Long-run asymmetry } & \multicolumn{3}{|c|}{ Short-run asymmetry } \\
\hline & $W_{L R}(R I P)$ & $W_{L R}(R)$ & $W_{L R}(O P)$ & $W_{S R}(R I P)$ & $W_{S R}(I R)$ & $W_{S R}(O P)$ \\
\hline \multicolumn{7}{|c|}{ Oil Importing } \\
\hline Austria & $\begin{array}{c}-3.2026^{* *} \\
(0.0454)\end{array}$ & $\begin{array}{c}-0.5843 * * * \\
(0.0058)\end{array}$ & $\begin{array}{c}1.3975^{* * *} \\
(0.0001)\end{array}$ & $\begin{array}{l}-0.1637^{*} \\
(3.4494)\end{array}$ & $\begin{array}{c}-0.0298 * * * \\
(14.0934)\end{array}$ & $\begin{array}{c}0.07144 * * * \\
(0.0000)\end{array}$ \\
\hline Estonia & $\begin{array}{c}-2.4029 * * * \\
(0.0004)\end{array}$ & $\begin{array}{l}-0.0303 \\
(0.4864)\end{array}$ & $\begin{array}{c}0.9808^{* * *} \\
(0.0000)\end{array}$ & $\begin{array}{c}-0.2635^{* * *} \\
(0.0000)\end{array}$ & $\begin{array}{l}-0.0033 \\
(0.5215)\end{array}$ & $\begin{array}{c}0.1075^{* * *} \\
(0.0000)\end{array}$ \\
\hline France & $\begin{array}{c}0.7184 \\
(0.6135)\end{array}$ & $\begin{array}{l}-0.0733 \\
(0.1268)\end{array}$ & $\begin{array}{c}0.1214 \\
(0.6271)\end{array}$ & $\begin{array}{c}0.0328 \\
(0.6202)\end{array}$ & $\begin{array}{c}-0.0033 * * \\
(0.0382)\end{array}$ & $\begin{array}{c}0.0055 \\
(0.6139)\end{array}$ \\
\hline Germany & $\begin{array}{l}2.5820^{*} \\
(0.0896)\end{array}$ & $\begin{array}{l}-0.0319 \\
(0.7240)\end{array}$ & $\begin{array}{l}-0.1583 \\
(0.4365)\end{array}$ & $\begin{array}{c}0.1335^{* *} \\
(0.0345)\end{array}$ & $\begin{array}{l}-0.0016 \\
(0.7117)\end{array}$ & $\begin{array}{c}-0.0081 \\
(0.4313)\end{array}$ \\
\hline Italy & $\begin{array}{c}0.5059 \\
(0.5917)\end{array}$ & $\begin{array}{l}-0.0545 \\
(0.1151)\end{array}$ & $\begin{array}{c}0.0742 \\
(0.6523)\end{array}$ & $\begin{array}{c}0.0289 \\
(0.5714)\end{array}$ & $\begin{array}{l}-0.0031 \\
(0.1420)\end{array}$ & $\begin{array}{c}-0.9874 * * * \\
(0.0006)\end{array}$ \\
\hline Japan & $\begin{array}{l}-0.3530 \\
(0.4690)\end{array}$ & $\begin{array}{c}-2.5400 * * * \\
(0.0004)\end{array}$ & $\begin{array}{c}0.5979 * * * \\
(0.0020)\end{array}$ & $\begin{array}{c}-.0444 \\
(0.4726)\end{array}$ & $\begin{array}{c}-0.3233 * * * \\
(0.0003)\end{array}$ & $\begin{array}{c}0.0752^{* * *} \\
(0.0015)\end{array}$ \\
\hline $\begin{array}{l}\text { New } \\
\text { Zealand }\end{array}$ & $\begin{array}{c}0.7174 \\
(0.2132)\end{array}$ & $\begin{array}{c}-0.0678^{*} \\
(0.0643)\end{array}$ & $\begin{array}{l}-0.0385 \\
(0.8667)\end{array}$ & $\begin{array}{c}0.0437 \\
(0.2573)\end{array}$ & $\begin{array}{l}-0 . \\
(0 .\end{array}$ & $\begin{array}{c}-0.0023 \\
(0.8679)\end{array}$ \\
\hline $\begin{array}{l}\text { South } \\
\text { Korea }\end{array}$ & $\begin{array}{l}-0.4568 \\
(0.7576)\end{array}$ & $\begin{array}{c}-0.0781^{* * *} \\
(0.0027)\end{array}$ & $\begin{array}{c}0.5406 \\
(0.3031)\end{array}$ & $\begin{array}{l}-0.0185 \\
(0.7567)\end{array}$ & $\begin{array}{c}-0.0031^{* * *} \\
(0.0030)\end{array}$ & $\begin{array}{c}0.0219 \\
(0.2821)\end{array}$ \\
\hline US & $\begin{array}{c}-1.2451^{* *} \\
(0.0218)\end{array}$ & $\begin{array}{c}-0.0412^{* *} \\
(0.0229)\end{array}$ & $\begin{array}{c}0.3690 * * * \\
(0.0000)\end{array}$ & $\begin{array}{c}-0.1494 * * \\
(0.0371)\end{array}$ & $\begin{array}{c}-0.0049 * * \\
(0.0263)\end{array}$ & $\begin{array}{c}0.0443 * * * \\
(0.0002)\end{array}$ \\
\hline \multicolumn{7}{|c|}{ Oil Exporting } \\
\hline Denmark & $\begin{array}{l}-0,6926 \\
(0.7899)\end{array}$ & $\begin{array}{l}-0.0465 \\
(0.3981)\end{array}$ & $\begin{array}{c}0,0052 \\
(0.9938)\end{array}$ & $\begin{array}{l}-0.0125 \\
(0.7655)\end{array}$ & $\begin{array}{c}-0.0008 \\
(0.3387)\end{array}$ & $\begin{array}{c}0.0009 \\
(0.9938)\end{array}$ \\
\hline Mexico & $\begin{array}{l}-0.9743 \\
(0.3168)\end{array}$ & $\begin{array}{c}0.0238 \\
(0.2915)\end{array}$ & $\begin{array}{c}0.2119 \\
(0.5652)\end{array}$ & $\begin{array}{l}-0.0524 \\
(0.3449)\end{array}$ & $\begin{array}{c}0.0012 \\
(0.1282)\end{array}$ & $\begin{array}{c}0.0114 \\
(0.6043)\end{array}$ \\
\hline Poland & $\begin{array}{c}-2.0513^{*} \\
(0.0566)\end{array}$ & $\begin{array}{c}-0.0680 * * * \\
(0.0043)\end{array}$ & $\begin{array}{c}0.9520 * * \\
(0.0144)\end{array}$ & $\begin{array}{l}-0.2326^{*} \\
(0.0672)\end{array}$ & $\begin{array}{c}-0.0077 * * * \\
(0.0017)\end{array}$ & $\begin{array}{c}0.1079 * * \\
(0.0169)\end{array}$ \\
\hline Portugal & $\begin{array}{c}0.0058 \\
(0.9972)\end{array}$ & $\begin{array}{c}-0.0719 \\
(0.3220)\end{array}$ & $\begin{array}{c}0.1224 \\
(0.7771)\end{array}$ & $\begin{array}{c}0.001 \\
(0.9972)\end{array}$ & $\begin{array}{c}-0.0018 \\
(0.2232)\end{array}$ & $\begin{array}{c}-0.0031 \\
(0.7826)\end{array}$ \\
\hline
\end{tabular}




\section{B. World prices}

\begin{tabular}{|c|c|c|c|c|c|c|}
\hline \multirow[b]{2}{*}{ Countries } & \multicolumn{3}{|c|}{ Long-run asymmetry } & \multicolumn{3}{|c|}{ Short-run asymmetry } \\
\hline & $W_{L R}(R I P)$ & $W_{L R}(R)$ & $W_{L R}(O P)$ & $W_{S R}(R I P)$ & $W_{S R}(I R)$ & $W_{S R}(O P)$ \\
\hline \multicolumn{7}{|c|}{ Oil Importing } \\
\hline Austria & $\begin{array}{l}-1.1174 \\
(0.5782)\end{array}$ & $\begin{array}{c}-0.9741 * * * \\
(0.0089)\end{array}$ & $\begin{array}{l}0.9590 * * \\
(0.0292)\end{array}$ & $\begin{array}{l}-0.0453 \\
(0.5859)\end{array}$ & $\begin{array}{c}-0.0395^{* * *} \\
(0.0001)\end{array}$ & $\begin{array}{c}0.0389 * * \\
(0.0111)\end{array}$ \\
\hline Estonia & $\begin{array}{l}-5.0908^{*} \\
(0.0600)\end{array}$ & $\begin{array}{c}0.0602 \\
(0.5896)\end{array}$ & $\begin{array}{l}1.6105 * * \\
(0.0350)\end{array}$ & $\begin{array}{c}-0.2948 * * \\
(0.0012)\end{array}$ & $\begin{array}{c}0.0034 \\
(0.5002)\end{array}$ & $\begin{array}{c}0.0301 * * * \\
(0.0023)\end{array}$ \\
\hline France & $\begin{array}{l}-1.9692 \\
(0.4577)\end{array}$ & $\begin{array}{c}-0.0511 \\
(0.2692)\end{array}$ & $\begin{array}{c}0.3249 \\
(0.4235)\end{array}$ & $\begin{array}{l}-0.0746 \\
(0.3931)\end{array}$ & $\begin{array}{l}-0.0019 \\
(0.1706)\end{array}$ & $\begin{array}{c}0.0123 \\
(0.3684)\end{array}$ \\
\hline Germany & $\begin{array}{c}2.7024 \\
(0.2476)\end{array}$ & $\begin{array}{l}-0.0485 \\
(0.6308)\end{array}$ & $\begin{array}{l}-0.1417 \\
(0.6934)\end{array}$ & $\begin{array}{c}0.1123 \\
(0.2035)\end{array}$ & $\begin{array}{l}-0.0020 \\
(0.6147)\end{array}$ & $\begin{array}{l}-0.0058 \\
(0.6913)\end{array}$ \\
\hline Italy & $\begin{array}{c}1.7709 \\
(0.4232)\end{array}$ & $\begin{array}{c}0.0240 \\
(0.6969)\end{array}$ & $\begin{array}{l}-0.4752 \\
(0.3233)\end{array}$ & $\begin{array}{c}0.0606 \\
(0.3275)\end{array}$ & $\begin{array}{c}0.0008 \\
(0.6749)\end{array}$ & $\begin{array}{l}-0.0162 \\
(0.1749)\end{array}$ \\
\hline Japan & $\begin{array}{l}-0.3667 \\
(0.2253)\end{array}$ & $\begin{array}{c}-2.1551^{* * *} \\
(0.0001)\end{array}$ & $\begin{array}{c}0.6220 * * * \\
(0.0000)\end{array}$ & $\begin{array}{c}-0.0662 \\
(0.2257)\end{array}$ & $\begin{array}{c}-0.3893 * * * \\
(0.0001)\end{array}$ & $\begin{array}{c}0.1123 * * * \\
(0.0000)\end{array}$ \\
\hline $\begin{array}{l}\text { New } \\
\text { Zealand }\end{array}$ & $\begin{array}{c}2.0967 \\
(0.1295)\end{array}$ & $\begin{array}{l}-0.0996 \\
(0.1971)\end{array}$ & $\begin{array}{l}-0.3355 \\
(0.4685)\end{array}$ & $\begin{array}{c}0.0708 \\
(0.1432)\end{array}$ & $\begin{array}{l}-0.0033 \\
(0.1392)\end{array}$ & $\begin{array}{l}-0.0113 \\
(0.4720)\end{array}$ \\
\hline $\begin{array}{l}\text { South } \\
\text { Korea }\end{array}$ & $\begin{array}{c}-0.4502 \\
(0.7249)\end{array}$ & $\begin{array}{c}-0.0551^{* *} \\
(0.0378)\end{array}$ & $\begin{array}{c}0.4270 \\
(0.3272)\end{array}$ & $\begin{array}{c}-0.0247 \\
(0.7284)\end{array}$ & $\begin{array}{c}-0.0030^{* *} \\
(0.0233)\end{array}$ & $\begin{array}{c}0.0234 \\
(0.3321)\end{array}$ \\
\hline US & $\begin{array}{c}-1.2454^{* *} \\
(0.0218)\end{array}$ & $\begin{array}{c}-0.0412^{* *} \\
(0.0229)\end{array}$ & $\begin{array}{c}0.3690 * * * \\
(0.0000)\end{array}$ & $\begin{array}{c}-0.1494^{* *} \\
(0.0371)\end{array}$ & $\begin{array}{c}-0.0049^{* *} \\
(0.0263)\end{array}$ & $\begin{array}{c}0.0443 * * * \\
(0.0002)\end{array}$ \\
\hline \multicolumn{7}{|c|}{ Oil Exporting } \\
\hline Denmark & $\begin{array}{c}4.1779 \\
(0.2907)\end{array}$ & $\begin{array}{l}-0.0403 \\
(0.4313)\end{array}$ & $\begin{array}{l}-1.5120 \\
(0.3142)\end{array}$ & $\begin{array}{c}0.07161 \\
(0.1085)\end{array}$ & $\begin{array}{c}-0.0006 \\
(0.3862)\end{array}$ & $\begin{array}{l}-0.0259 * \\
(0.0554)\end{array}$ \\
\hline Mexico & $\begin{array}{c}0.1194 \\
(0.9709)\end{array}$ & $\begin{array}{c}0.0696 \\
(0.4045)\end{array}$ & $\begin{array}{l}-0.4480 \\
(0.6971)\end{array}$ & $\begin{array}{c}0.0023 \\
(0.9707)\end{array}$ & $\begin{array}{c}0.0013^{* * *} \\
(0.0067)\end{array}$ & $\begin{array}{l}-0.0089 \\
(0.6153)\end{array}$ \\
\hline Poland & $\begin{array}{c}0.1716 \\
(0.8720)\end{array}$ & $\begin{array}{l}-0.0210 \\
(0.3030)\end{array}$ & $\begin{array}{l}-0.1065 \\
(0.7113)\end{array}$ & $\begin{array}{c}0.0190 \\
(0.8714)\end{array}$ & $\begin{array}{l}-0.0023 \\
(0.2750)\end{array}$ & $\begin{array}{l}-0.0118 \\
(0.7097)\end{array}$ \\
\hline Portugal & $\begin{array}{c}1.7593 \\
(0.3516)\end{array}$ & $\begin{array}{c}-0.0869 \\
(0.2032)\end{array}$ & $\begin{array}{l}-0.6035 \\
(0.2146)\end{array}$ & $\begin{array}{c}0.0526 \\
(0.3680)\end{array}$ & $\begin{array}{l}-0.0026 \\
(0.1110)\end{array}$ & $\begin{array}{l}-0.0180 \\
(0.2079)\end{array}$ \\
\hline
\end{tabular}

(Note) This table reports the results of the long- and short-run symmetry tests for the effect of each explanatory variable (real industrial production, short term interest rate and real oil price) on stock price. $W_{L R}$ denotes the Wald statistic for the long-run symmetry, which tests the null hypothesis of $\theta^{+}=\theta^{-}$for each explanatory variable in Equation (4). $W_{S R}$ corresponds to the Wald statistic for the short-run asymmetry, which tests the null hypothesis that $\pi_{i}^{+}=\pi_{i}^{-}$for each explanatory variable in Equation (4). The numbers in brackets are the associated $p$-values. ${ }^{*}, * *$ and $* * *$ indicate rejection of the null hypothesis of symmetry at the $10 \%, 5 \%$ and $1 \%$ levels, respectively. 
Table 6 shows the estimated results of the NARDL model. Based on these results, we note that the estimated coefficients related to the short-term interest rates are highly significant at the $1 \%$ and $5 \%$ significant levels for almost all the selected countries. For oil prices, the significance of the coefficients seems to depend on the economic state of each country. The same results are confirmed for the coefficients related to real industrial production. Based on the AIC information criterion and Wald symmetry tests, we selected the NARDL lag specification with the short-run asymmetry for each country. The results show that the long-run coefficient of positive changes in oil prices is positive for the national oil price of New Zealand and the world oil prices of Austria. For Germany and the US, the long-run coefficient of the positive changes in both national and world oil prices is negative. For the negative changes in oil prices, the long-run coefficient is negative at the $1 \%$ and $5 \%$ significance levels for Estonia and Germany. For Japan and the US, the long-run coefficient of negative changes in oil prices is negative for both the national and the world oil price specification. For the short-term interest rate, both positive and negative changes present a negative long-run coefficient at the significance levels of $1 \%$ and $5 \%$ in Italy, Japan, South Korea, New Zealand, and Poland. In the US, positive changes in the short-term interest rate have a positive impact on stock prices. The long-run coefficient of negative changes in the short-term interest rate is negative for Austria. For real industrial production, both positive and negative changes present a positive long-run coefficient at the $1 \%$ and $5 \%$ significance levels for France, Italy, Japan, Mexico, Poland, and the US. For Estonia, the negative changes in real industrial production present a positive long-run coefficient. Only for Austria, the long-run coefficient is positive for the positive changes in real industrial production. 


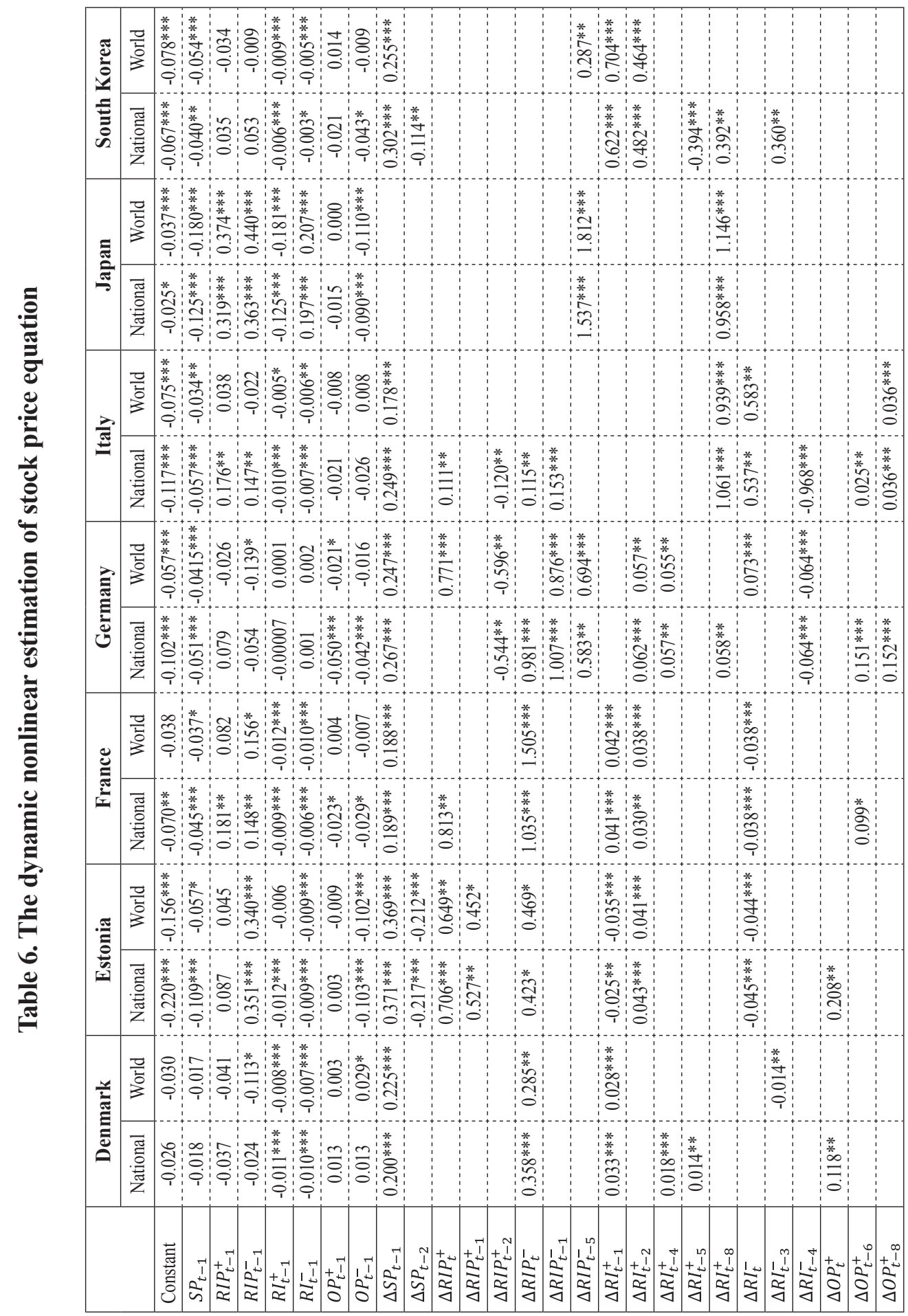




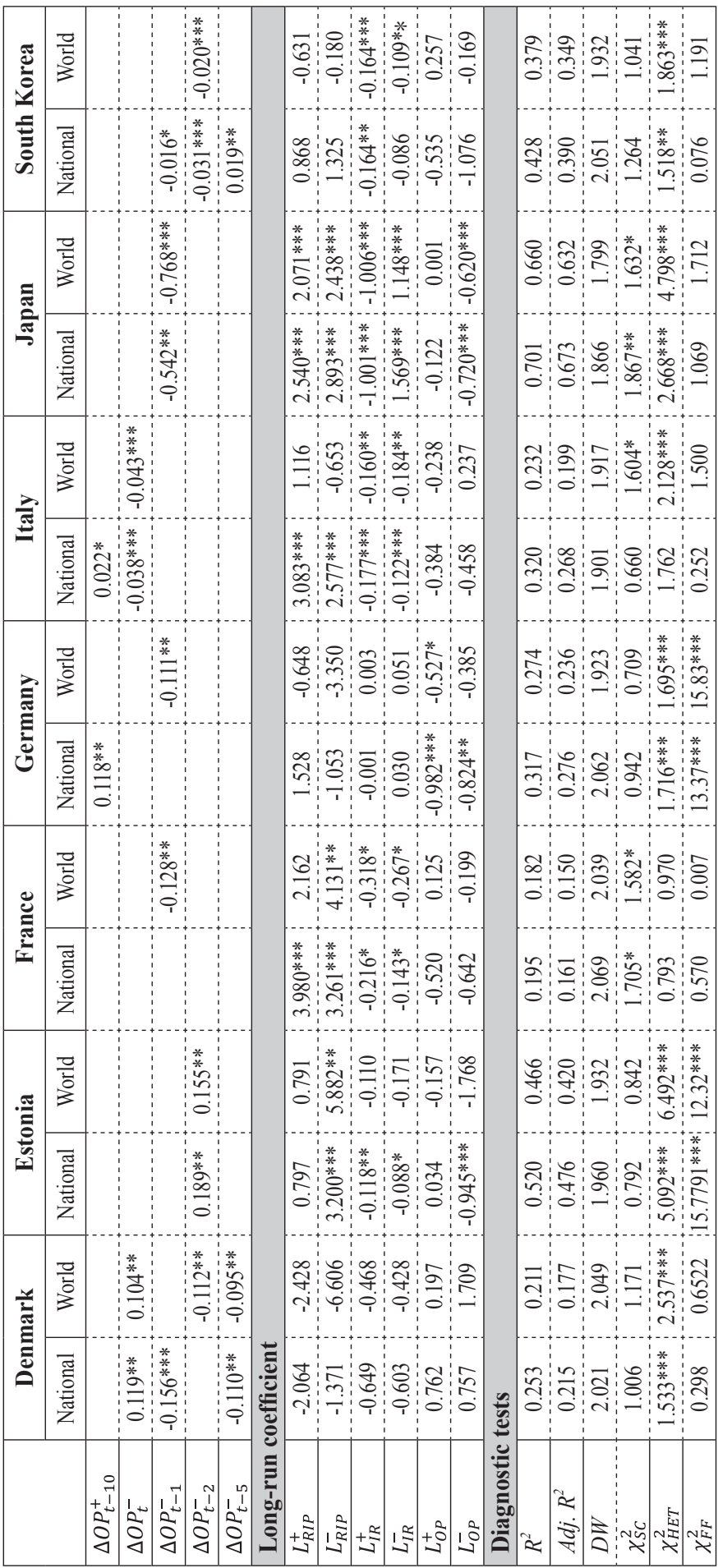




\begin{tabular}{|c|c|c|c|c|c|c|c|c|c|c|c|c|c|c|c|c|c|c|c|c|c|c|c|c|c|}
\hline 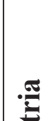 & 吾 & 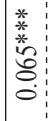 & 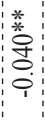 & $\begin{array}{l}\text { 㟟 } \\
0 \\
0\end{array}$ & $\frac{{ }_{\stackrel{*}{\circ}}^{\circ}}{2}$ & 离 & $\underset{\delta}{\delta}$ & 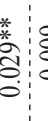 & \begin{tabular}{l}
8 \\
\hdashline \\
\hdashline
\end{tabular} & 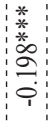 & 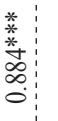 & 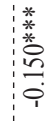 & 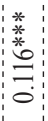 & & 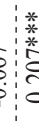 & 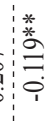 & 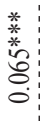 & 倍 & $\begin{array}{ll}* \\
* \\
\end{array}$ & 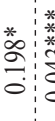 & 항 & : & 8 & $\frac{0}{6}$ & 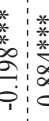 \\
\hline 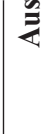 & 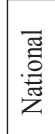 & $\stackrel{\infty}{\infty}$ & 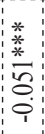 & $\left\{\begin{array}{l}n \\
0 \\
0 \\
1\end{array}\right.$ & $\frac{\infty}{0}$ & 8 & $\begin{array}{l}\widehat{8} \\
\bar{c}\end{array}$ & $\begin{array}{c}\bar{Z} \\
\bar{\delta} \\
\end{array}$ & $\begin{array}{l}0 \\
\end{array}$ & 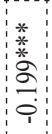 & 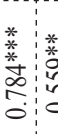 & 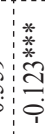 & 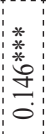 & \begin{tabular}{ll}
$*$ \\
\multirow{2}{*}{} \\
$o$ \\
$o$ \\
0
\end{tabular} & & & $\begin{array}{l}\infty \\
0 \\
0\end{array}$ & $\begin{array}{l}* \% \\
0 \\
0\end{array}$ & $\begin{array}{l}n \\
\stackrel{0}{0}\end{array}$ & $\begin{array}{c}0 \\
0 \\
0\end{array}$ & ه̊ & & $\frac{1}{4}$ & E & \\
\hline & 믈 & ợ & a & 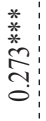 & 菨 & $\overline{8}$ & 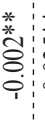 & 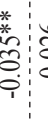 & :ִ & & * & & & & & & $\begin{array}{l}0 \\
0 \\
0 \\
0\end{array}$ & & & & & & & * & \\
\hline & 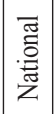 & 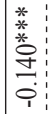 & \begin{tabular}{l}
$*$ \\
\multirow{2}{*}{} \\
0 \\
0
\end{tabular} & 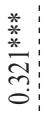 & â & 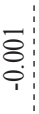 & i & $\frac{2}{E}$ & \pm & & & & & & & & $\begin{array}{l}\text { * } \\
\text { कo } \\
9 \\
9\end{array}$ & $\begin{array}{l}\text { \% } \\
\frac{*}{9}\end{array}$ & & 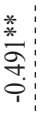 & & & & & \\
\hline
\end{tabular}

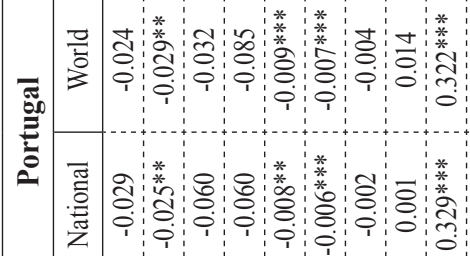

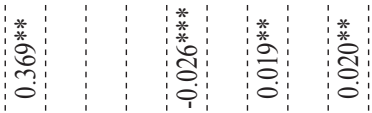

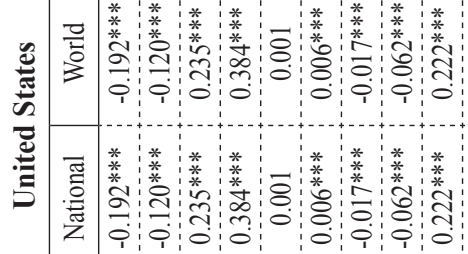

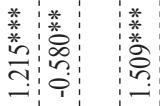

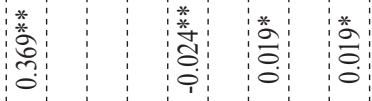

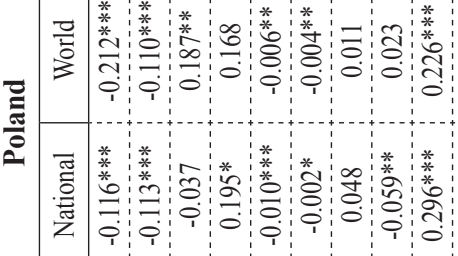

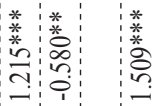

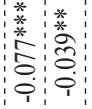

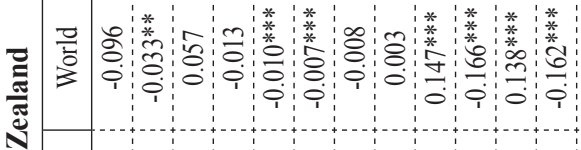

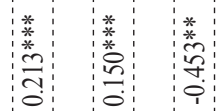

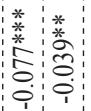

之.

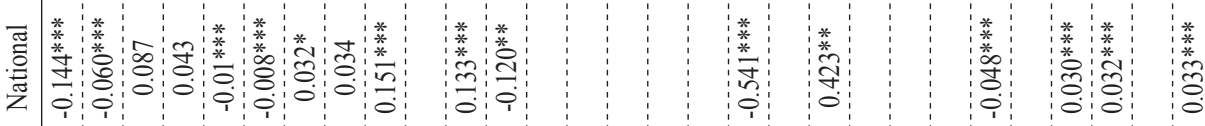


氖

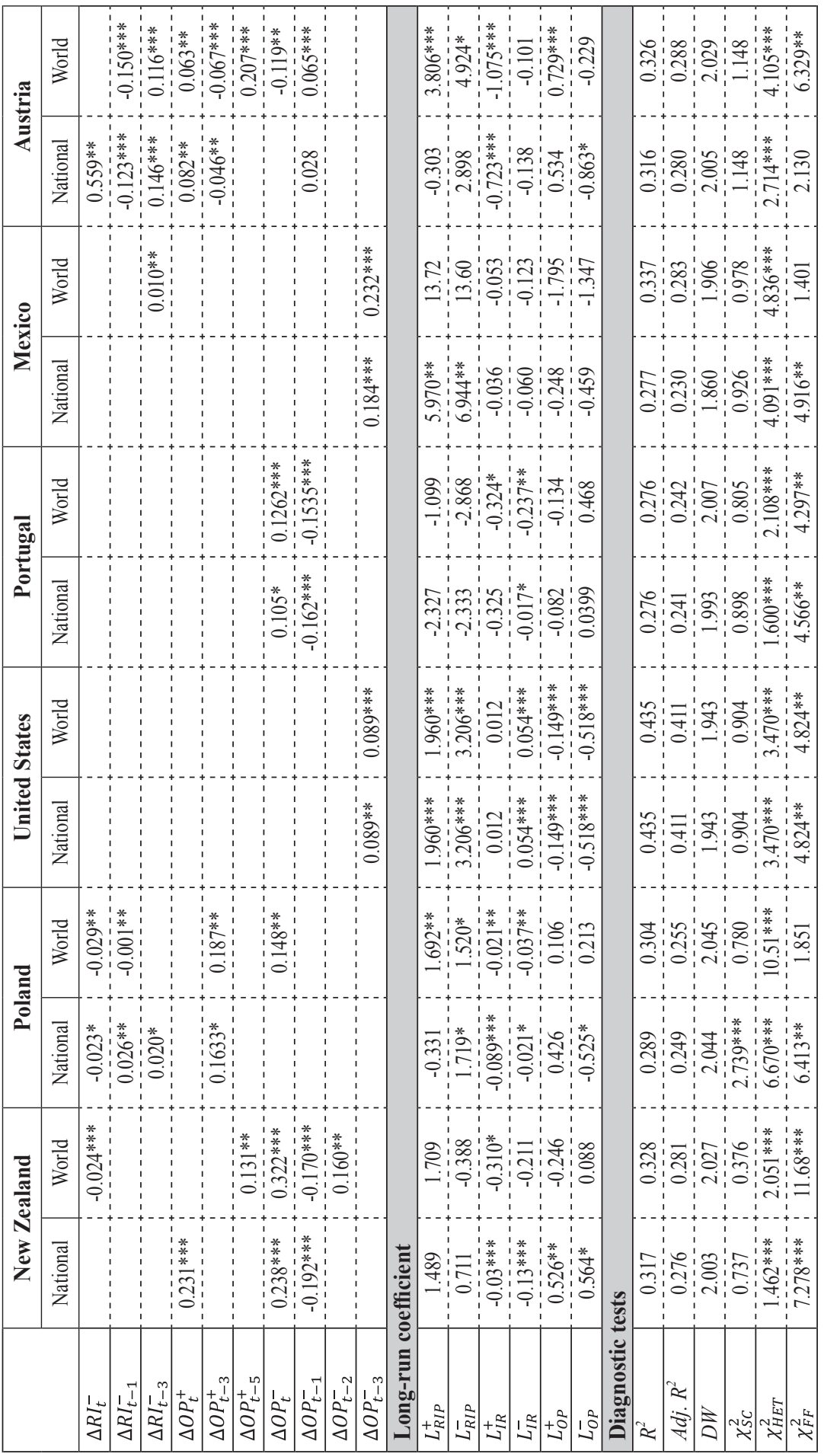

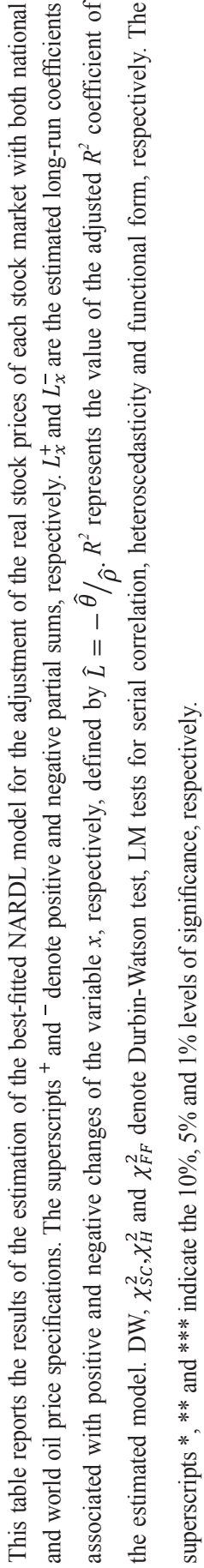

量 
Figures 1 and 2 plot the dynamic effects of positive and negative changes in real industrial production, short-term interest rates, and oil prices for the sampled countries, considering the national and world oil price specifications, respectively.

Based on Figures 1 and 2, an asymmetric response of stock prices to positive and negative changes in real industrial production is detected with a rapid reaction for the following economies: Germany, Italy, South Korea, Poland, the US, and Austria. For Germany, Poland, and Austria, we see a particularly insignificant reaction to positive changes and a negative and significant reaction to negative changes. For the US and Italy, the responses to decreases are obviously gradual, with a smooth equilibrium correction after about 5 to 6 months and 6 to 8 months, respectively. The reaction of stock prices to negative changes in real industrial production in Estonia is cumulative over time. Whereas, the reaction to positive shocks is absorbed after approximately 6 to 7 months to reach an equilibrium state over the rest of the months. For Japan and France, a rapid and quite symmetric reaction of stock prices to positive and negative shocks in real industrial production is detected. For Denmark, Mexico, and New Zealand, we cannot detect a significant rapid response to increases or decreases in real industrial production.

The results of the short-term interest rate show a mixed response of stock prices to positive and negative shocks. For Denmark and Japan, we cannot detect a rapid response of stock prices due to negative and positive shocks occurring in the shortterm interest rate. The reaction appears within approximately 9 months. In addition, an asymmetric reaction is detected in Estonia during the first 6 months. After this period, the response becomes symmetric but does not adjust to the equilibrium state. For France, both positive and negative shocks in the short-term interest rate positively affect the stock market prices. However, although the sensitivity to negative shocks continues over time, the positive impact of an increase in the short-term interest rate diminishes after approximately 5 months to become negative in the ninth month. The reaction of the stock prices in Germany to the variation in the short-term interest rate is positive and more rapid to an increase (after approximately 4 months) than to a decrease (after approximately 6 months). In Italy and Austria, we detect an asymmetric reaction with an insignificant impact of increases and decreases, respectively, in the short-term interest rate. It is notable that the stock prices respond positively to negative shocks in the shortterm interest rate for Italy but negatively to increases for the Austrian economy. For South Korea and Mexico, we detect an asymmetric response. However, the reaction is more rapid in Mexico (after 1 to 2 months) than in South Korea (after approximately 5 to 6 months). The response to increases is negative and more gradual in South Korea, 
but it is positive and more gradual in Mexico. We also show asymmetry in the relation in the US, New Zealand, and Poland. For these countries, the response is nonlinear with a negative impact of increases over time but an unstable impact of decreases. In the US, a negative impact of increases and decreases is detected, but it is more gradual for the increases.

For both national and world oil prices, we note an asymmetric response of stock market prices to positive and negative shocks. However, although there is clear asymmetry, both positive and negative shocks in oil prices remain insignificant in Portugal for national oil prices and in France, Italy, and Portugal for world oil price specifications. Positive and negative shocks have a time-deferred impact for the rest of the countries. The empirical results discussed above have significant implications for investors, analysts, and political decision makers. First of all, we note that stock market prices are highly sensitive to positive and negative shocks occurring in the short-term interest rate, compared with changes in real industrial production and in oil prices. It is apparent that stock prices react immediately and strongly to changes in the shortterm interest rate. However, oil price shocks have an insignificant impact because the sample countries are highly industrialized and have a high GDP. Because the abrupt changes in oil prices are transmitted to the stock market through industrial production and short-term interest rates as indirect transmission channels to the interest rate, these countries adjust their policy on oil reserves to smoothen the impact on stock returns. The adjustment process also involves the central bank adjusting the interest rate policy to address the inflation rate induced by oil prices because oil prices act as an inflationary factor. The positive and negative shocks in the oil price effect become absorbed and recompensed by the loss and gain induced by this adjustment of the short-term interest rate. The impact of changes in oil prices on real industrial production is neutralized by the effect of adjustment of the short-term interest rate.

Overall, our findings confirm that stock prices are related in a nonlinear manner to macroeconomic fundamentals such as oil prices, real industrial production, and short-term interest rates. The response is highly sensitive to whether the changes in macroeconomic variables are positive or negative. In particular, the speed of response and the time required to reach a new equilibrium state are sensitive to the direction of changes in the macroeconomic fundamentals. 


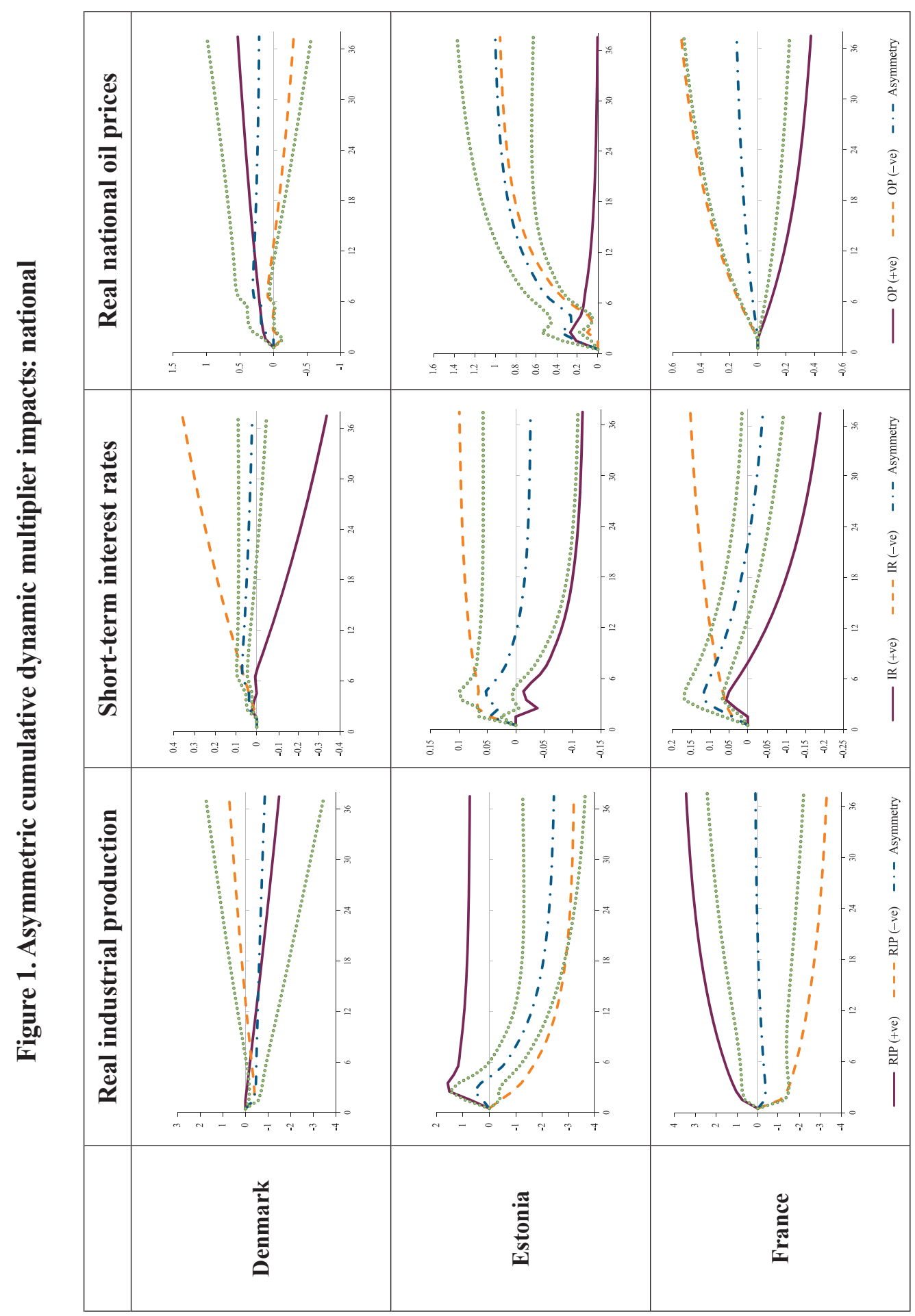




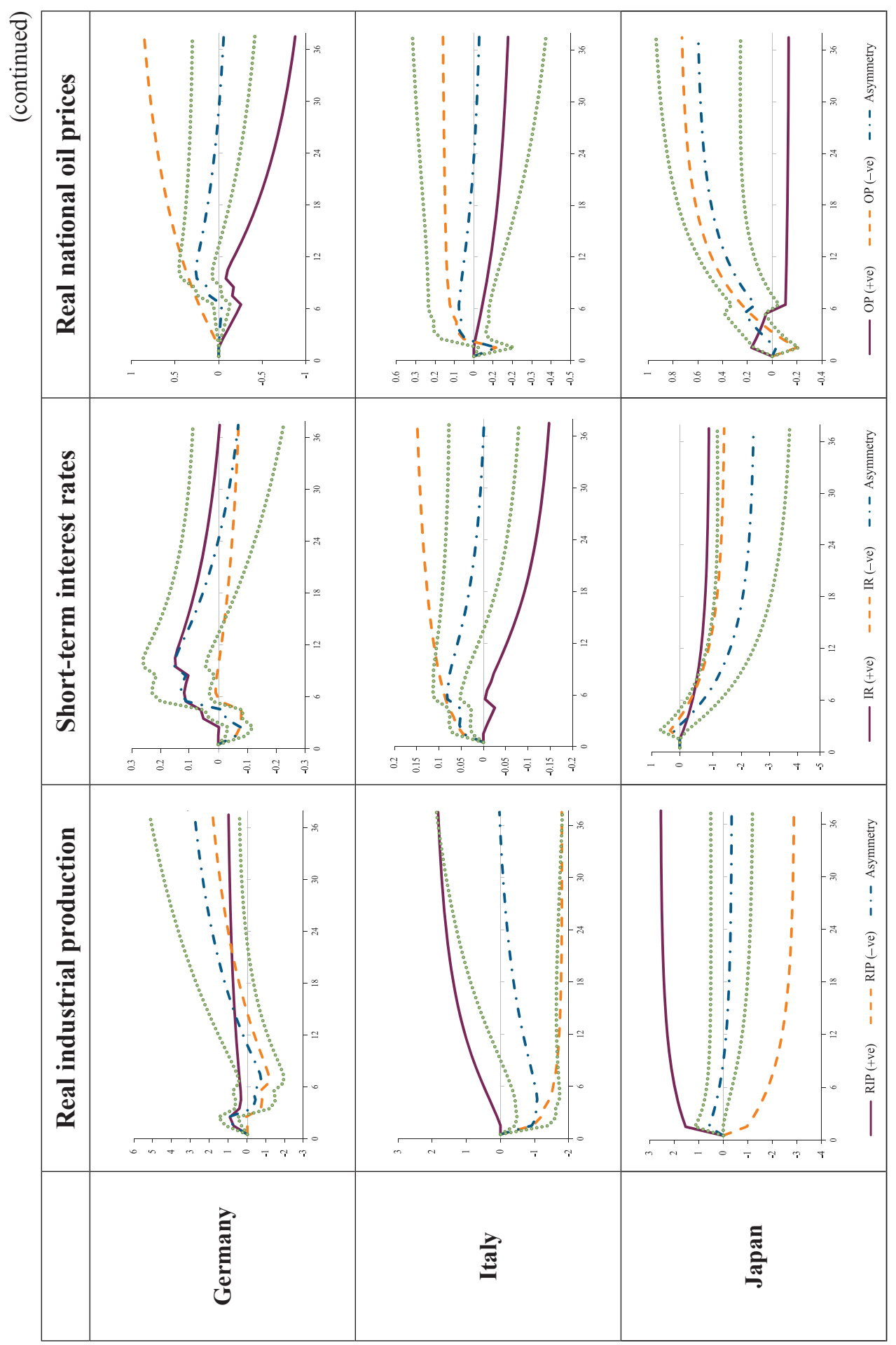




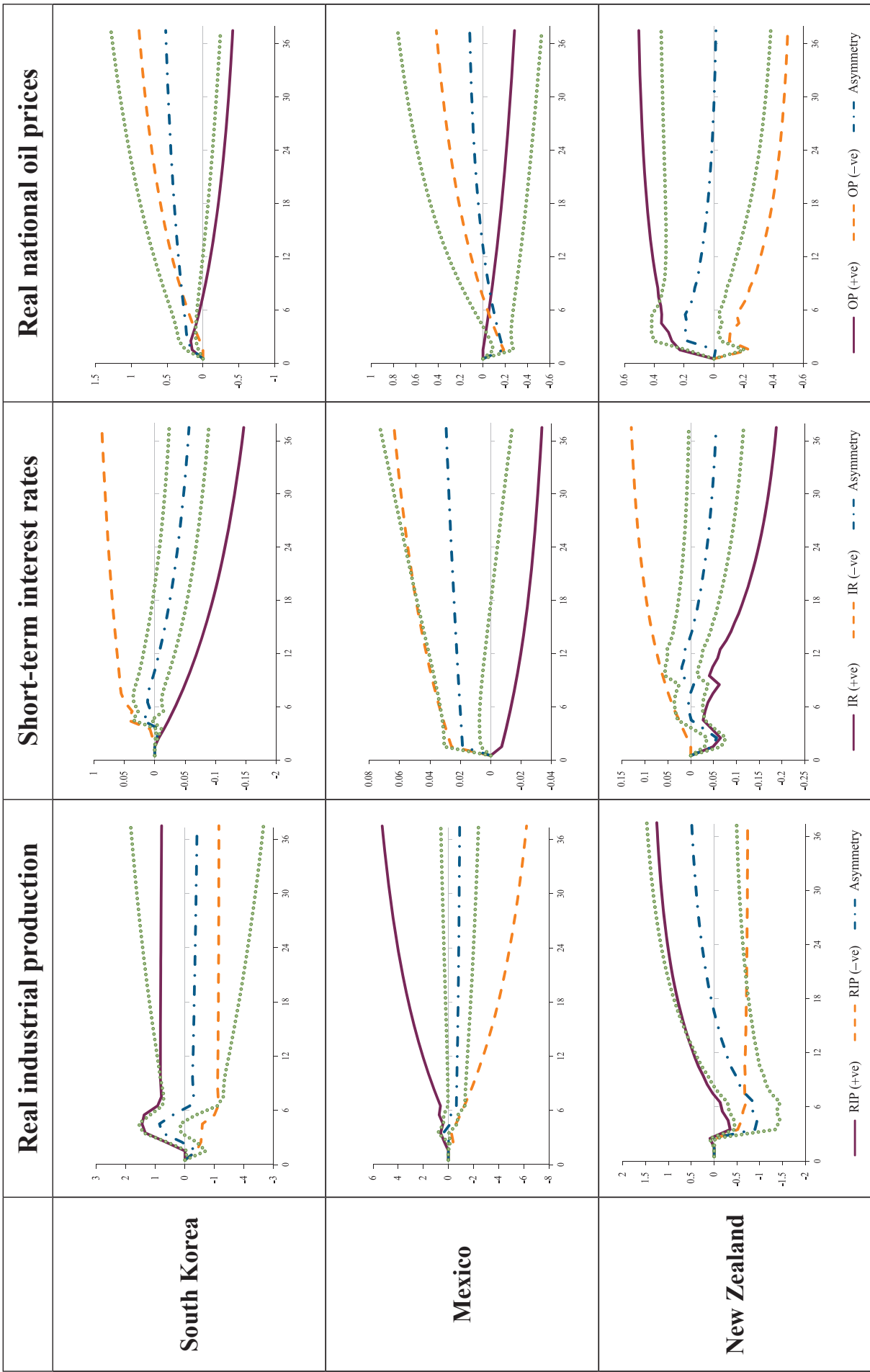




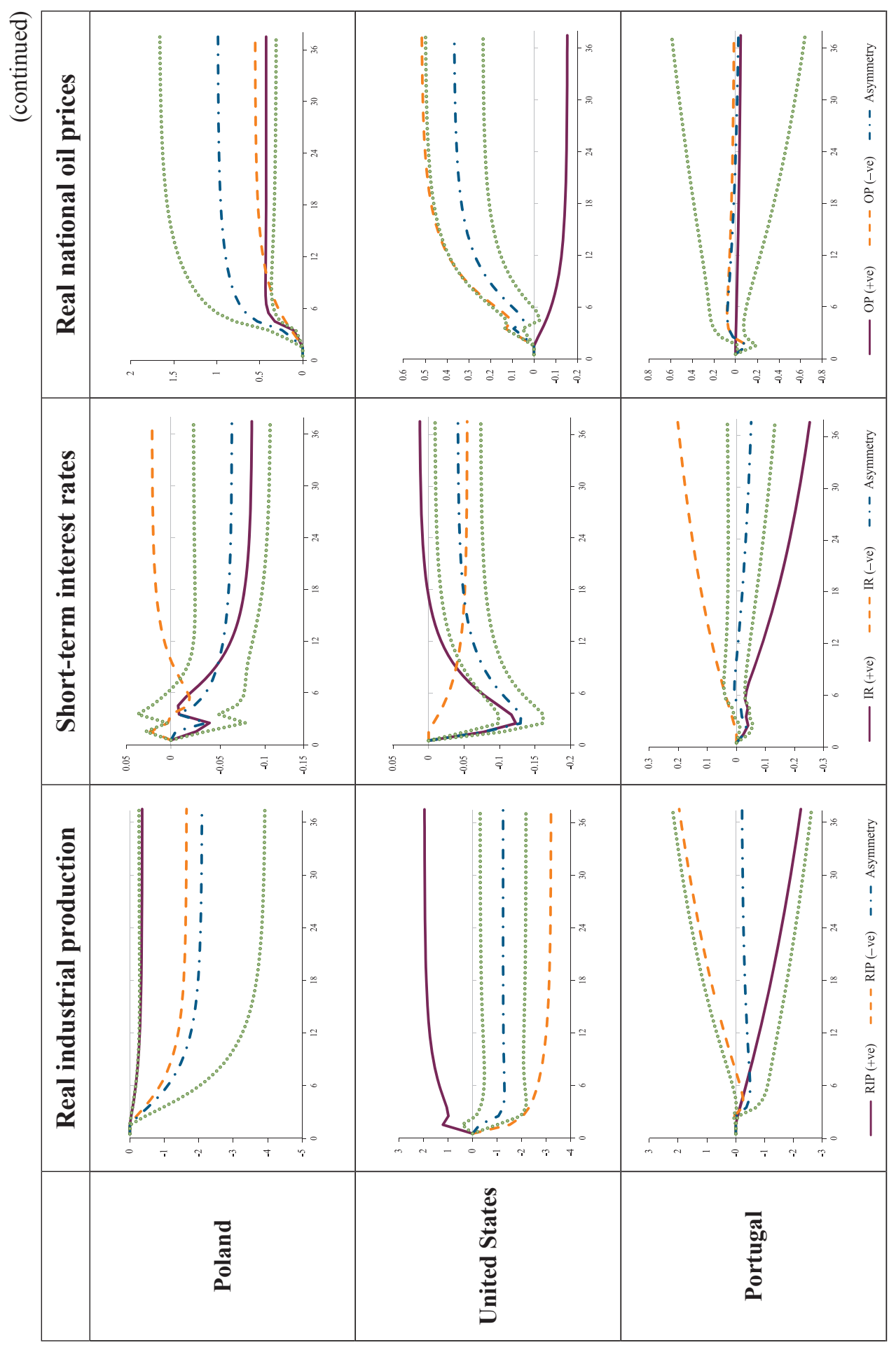




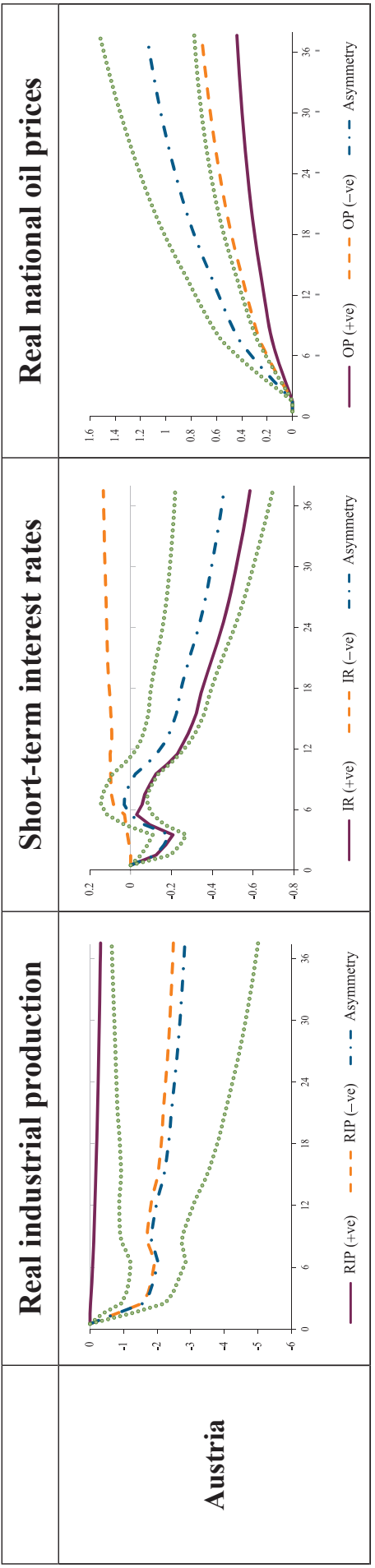

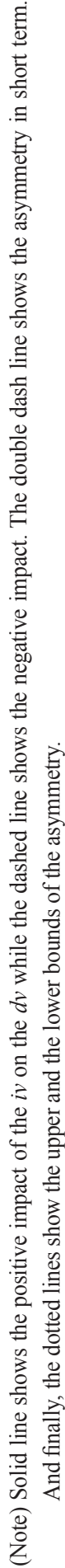




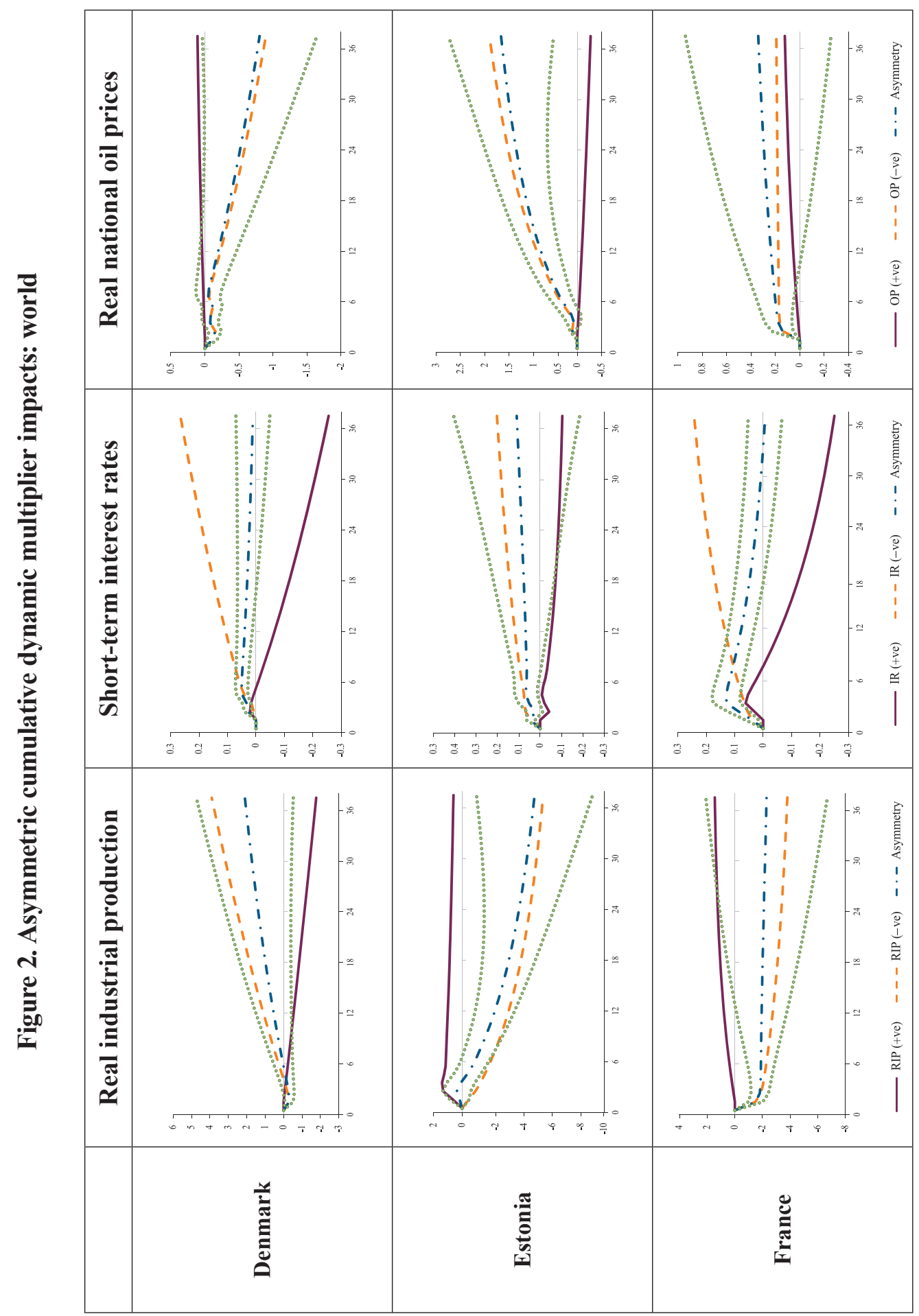




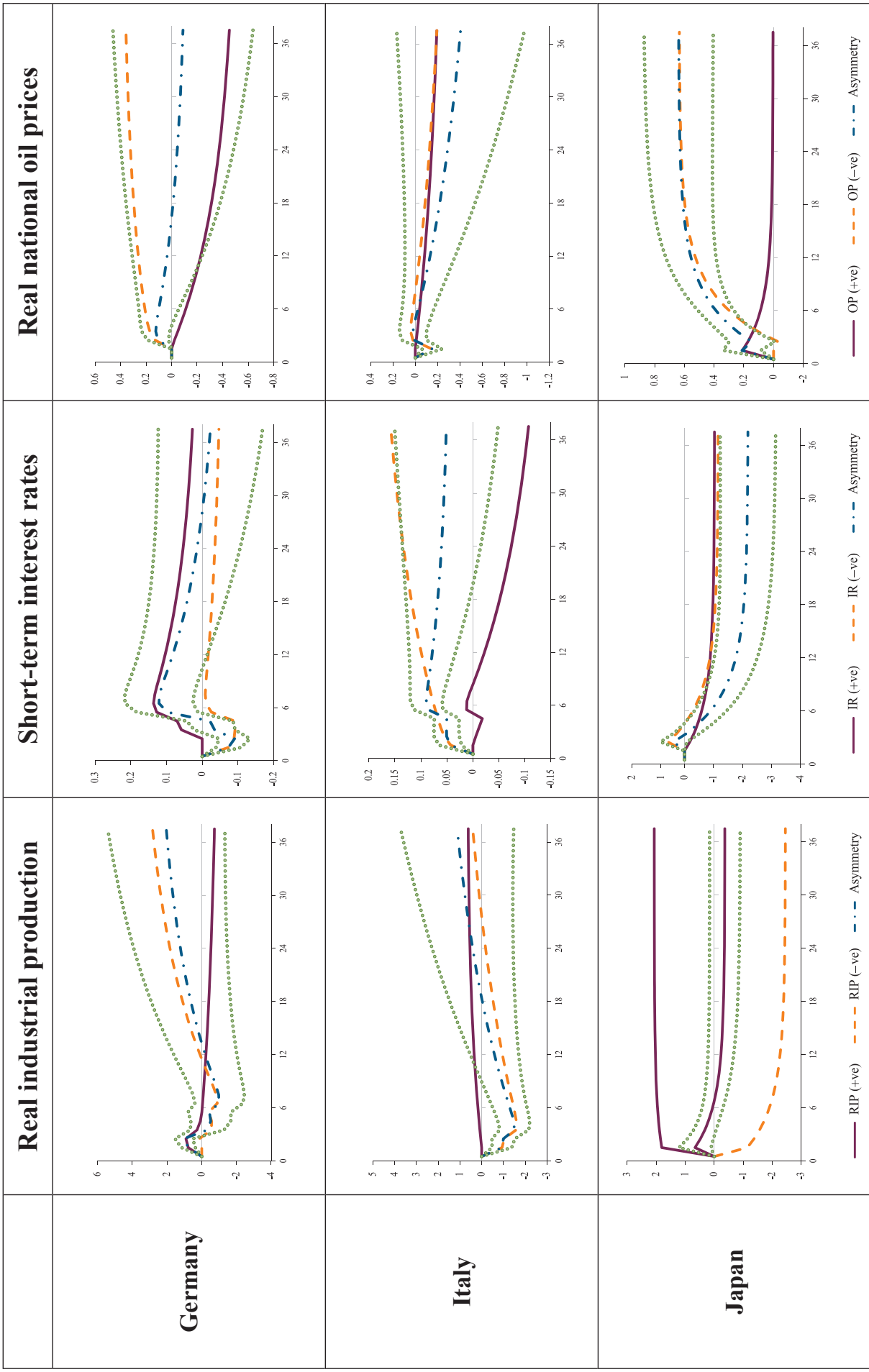




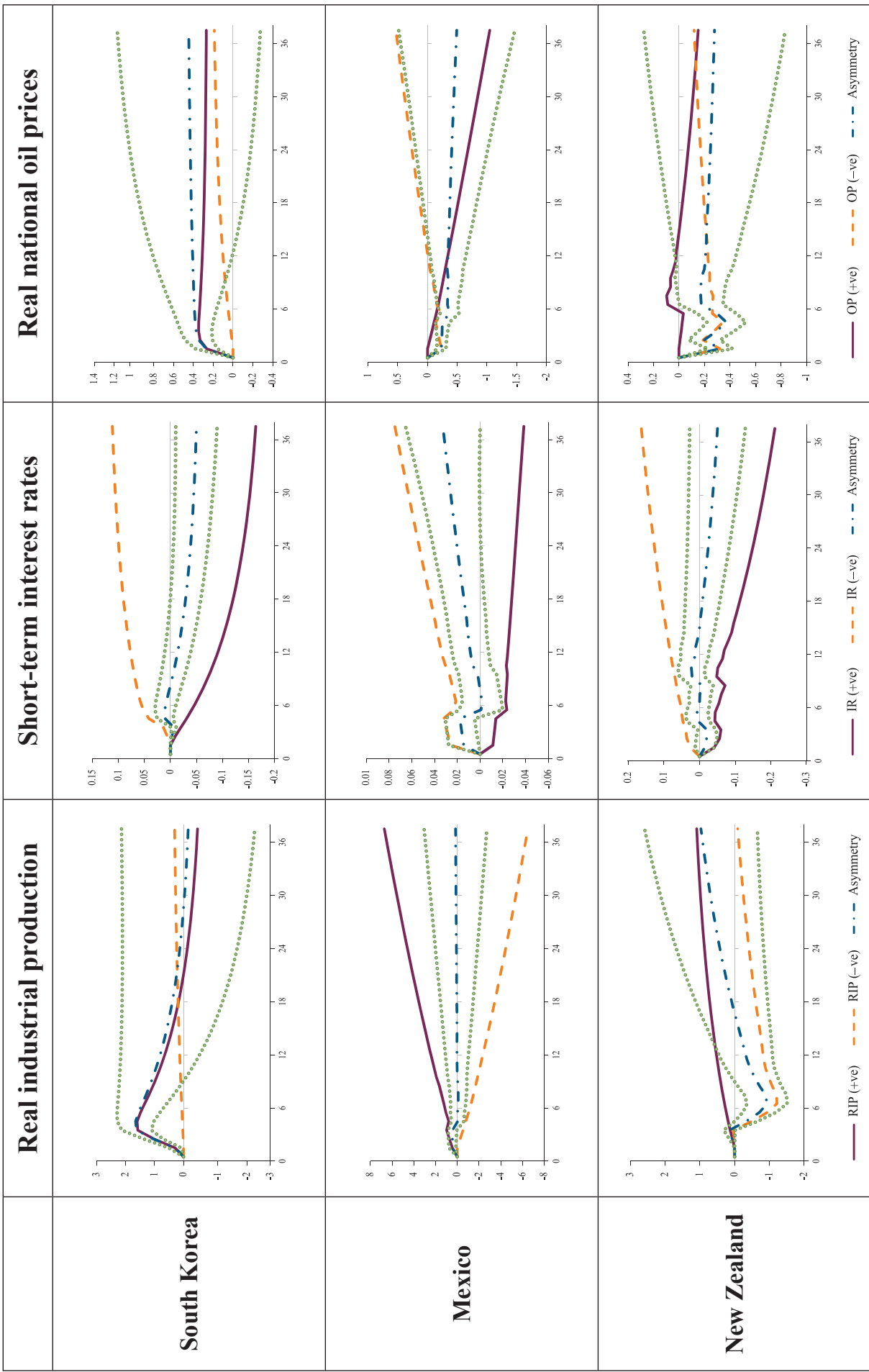




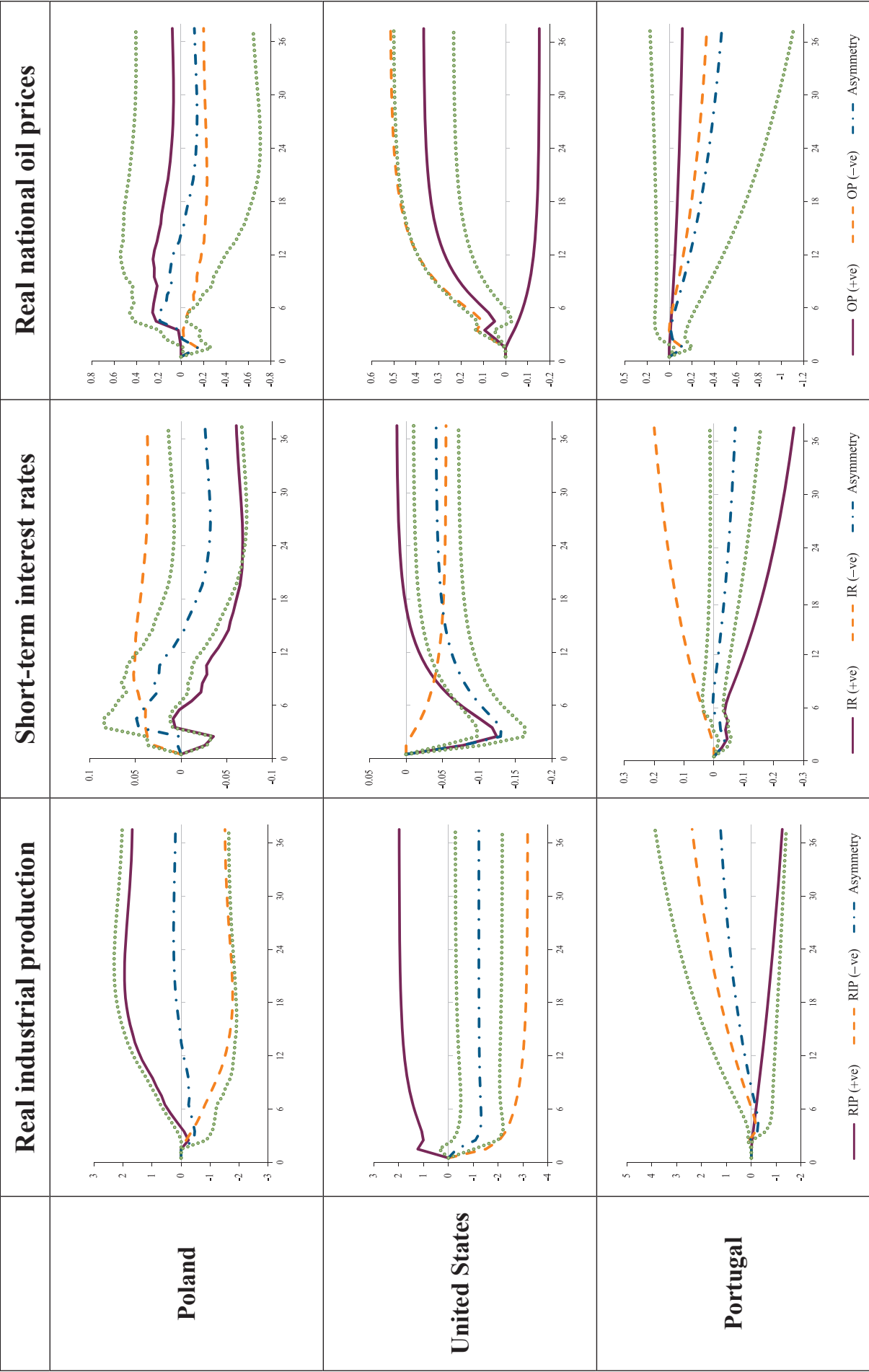




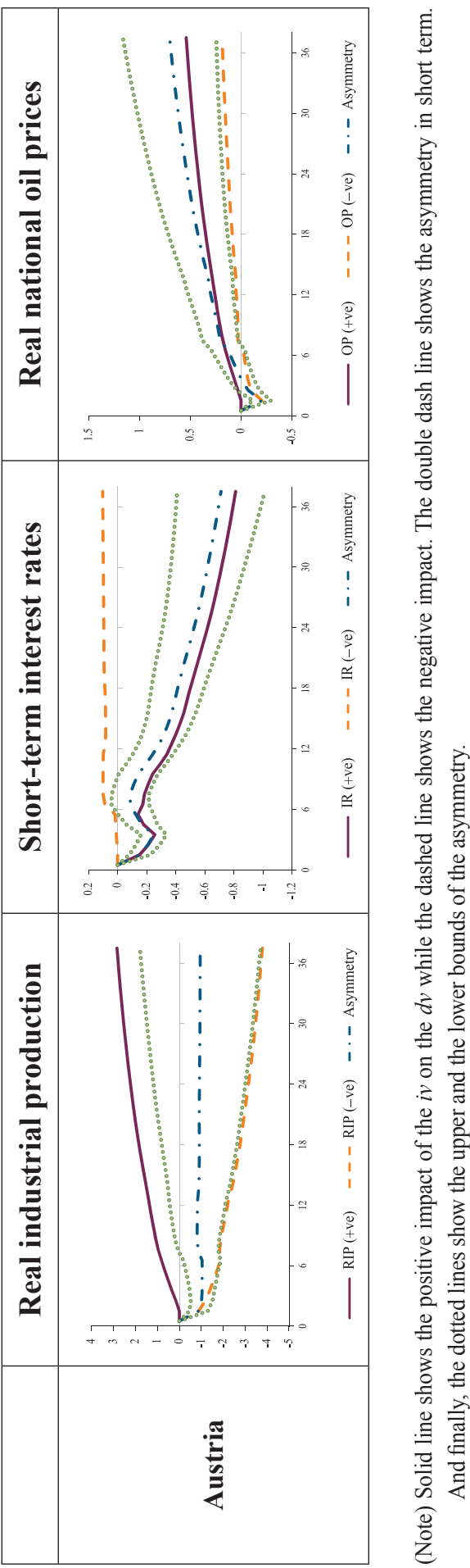




\section{Conclusions}

This paper investigates the dynamic asymmetric response of stock prices to oil prices, real industrial production, and short-term interest rates, using monthly data of OECD oil net importer and net exporter countries. The short- and long-run asymmetries in the relation are estimated using the NARDL model with monthly data from January 1986 to May 2015.

The results show an asymmetric long-run impact of oil prices, real industrial production, and short-term interest rates on the stock markets of Poland, the US and, Austria. For Estonia, only real industrial production and oil prices have an asymmetric impact on the stock market in the long run. For the stock market of Japan, the oil prices and short-term interest rate have an asymmetric impact. For the German and South Korean stock markets, the long-run asymmetry moves from real industrial production to stock markets and from short-term interest rates to stock markets, respectively.

Overall, our findings serve as confirmation that the stock market prices are related in a nonlinear way to macroeconomic fundamentals, such as oil prices, real industrial production, and short-term interest rates. The response is highly sensitive to whether the changes in macroeconomic variables are positive or negative. In particular, the speed of response and the time required to reach a new equilibrium state are sensitive to the direction of the changes in the macroeconomic fundamental. This finding can substantially help investors to adjust their investment strategies to these changes, and it lends itself to more efficient decision-making policies because it can help investors smoothen the negative impact of unexpected events.

Received 8 December 2017, Revised 5 January 2018, Accepted 9 February 2018 


\section{References}

Antoniou, A., Holmes, P., Priestley, R., The effects of stock index futures trading on stock index volatility: An analysis of the asymmetric response of volatility to news. Journal of Futures Markets 18(2) (1998): 151-166.

Arouri, M., Lahiani, A., Nguyen, K. D., Return and volatility transmission between oil prices and stock markets of the GCC countries.Economic Modelling 28 (2011): 18151825.

Awartani , B., Maghyereh, A. I., Dynamic spillovers between oil and stock markets in the Gulf Cooperation Council Countries.Energy Economics 36 (2013): 28-42.

Aye, G.C., Does oil price uncertainty matter for stock returns in South Africa?. Investment Management and Financial Innovations 12(1) (2015): 179-187.

Bahng, J.S., Shin, S., Do stock price indices respond asymmetrically? Evidence from China.Japan and South Korea.Journal of Asian Economics 14 (2003): 541-563.

Basher, S., Haug, A., Sadorsky, P., Oil prices, exchange rates and emerging stock markets. Energy Economics 34 (2012): 227-240.

Başkaya, Y.S., Hülagü, T. Kücük, H., Oil price uncertainty in small open economy. IMF Economic Review 61(1) (2013): 168-198.

Bernanke, B.S., Gertler, M., Watson, M.W., Systematic monetary policy and the effects of oil price shocks. Brookings Papers on Economic Activity 1 (1997): 91-157.

Blanchard, O.J., Gali, J., The macroeconomic effects of oil price shocks: why are the 2000s so diffrent from the 1970s? In: Gali. J. and Gertler M.J. (eds).International Dimensions of Monetary Policy. Chicago: University of Chicago Press, (2009): 373421. 
Bouri, E., A broadened causality in variance approach to assess the risk dynamics between crude oil prices and the Jordanian stock market.Energy Policy 85 (2015): 271-279.

Caporale, G.M., Ali, F.M., Spagnolo, N., Oil price uncertainty and sectoral stock returns in China: A time-varying approach. China Economic Review 34 (2015): 311321.

Chen, N., Roll, R., Ross, S.A., Economic forces and the stock market.Journal of Business 59(3) (1986): 383-403.

Cologni, A., Manera, M., The asymmetric effects of oil shocks on output growth: a Markov-switching analysis for the G-7 Countries. Economic Modelling 26 (2009): $1-29$.

Cũnado, J., Jo, S., Perez de Gracia, F., Revising the macroeconomic impact of oil shocks in Asian economies. Bank of Canada, working paper (2015): 2015-2023.

Cũnado, J., Perez de Gracia, F., Oil price shocks and stock market returns: evidence for some European countries. Energy Economics 42 (2014): 365-377.

Cũnado, J., Perez de Gracia, F., Do oil price shocks matter? Evidence for some European countries.Energy Economics 25 (2003): 137-154.

Cũnado, J., Perez de Gracia, F., Oil prices, economic activity and inflation: evidence for some Asian economies. Quarterly Review of Economics and Finance 45(1) (2005): 65-83.

Degiannakis, S., Filis., G., and Floros., C., Oil and stock returns: Evidence from European industrial sector indices in a time-varying environment. Journal of International Financial Markets Institutions and Money 26 (2003): 175-191. 
Dhaoui, A., Saidi, Y., 2015. Oil supply and demand shocks and stock price: empirical evidence for some OECD countries. MPRA Paper No. 63556.

Driesprong, G., Jacobsen, B., Maat, B., Striking oil: another puzzle? Journal of Financial Economics 89(2) (2008): 307-327.

Edelstein, P., Kilian, L., The response of business fixed investment to energy price changes: A test of some hypotheses about the transmission of energy price shocks. The B.E. Journal of Macroeconomics 7 (2007): 1-39.

El-Sharif, I., Brown, D., Burton, B., Nixon, B., Russell, A., Evidence on the nature and extent of the relationship between oil prices and equity values in the UK.Energy Economics 27(6) (2005): 819-830.

Engemann, K.M., Kliesen, K.L., Owyang, M.T., Do oil shocks drive business cycle? Some US and international evidence.Macroeconomic Dynamics 15 (2011): 298-517.

Faff, R., Brailsford, T., Oil price risk and the Australian stock market.Journal of Energy Finance and Development, 4(1) (1999): 69-87

Filis, G., Degiannakis, S., Floros, C., Dynamic correlation between stock market and oil prices: The case of oil-importing and oil-exporting countries. International Review of Financial Analysis 20(3) (2011): 152-164.

French, K.R.C., Schwert, G.W., Stambaugh, R.F., Expected stock returns and volatility. Journal of Financial Economics 3.(1987): 3-29.

Gogineni, S., Oil and the stock market: An industry level analysis. Financial Review 45. (2010): 995-1010.

Hamilton, J.D., Oil and the macroeconomy since World War II.The Journal of Political Economy 91(2) (1983): 228-248. 
Hamilton, J.D., This is what happened to the oil price-macroeconomy relationship. Journal of Monetary Economics 38(2) (1996): 215-220.

Hamilton, J.D., What is an oil shock?Journal of Econometrics 113(2) (2003): 363398.

Hamilton, J.D., 2005. Oil and the macroeconomy. In: Durlauf.S..Blume.L. (Eds.).The New Palgrave Dictionary of Economics. $2^{\text {nd }}$ Edition. Macmillan. London.

Hamilton, J.D., Nonlinearities and the macroeconomic effects of oil prices. Macroeconomic Dynamics 15 (2011): 364-378.

Hamilton, J.D., Herrera, A., Oil shocks and aggregate macroeconomics behavior: The role of monetary policy. Journal of Money, Credit, and Banking 36(2) (2004): 265286

Huang, R.D., Masulis, R.W., Stoll, H.R., Energy shocks and financial markets.Journal of Futures Markets 16 (1996): 1-27.

Ibrahim, M.H., 2015. Oil and food prices in Malaysia: a nonlinear ARDL analysis. Agricultural and Food Economics 2(3), http://www.doi.org/10.1186/s40100-014-0020-3.

Jalil, N.A., Ghani, G.M., Duasa, J., Oil price and the Malaysia Economy.International Review of Business Research Papers 5(4) (2009): 232-256.

Jones, C.M., Kaul, G., Oil and the stock markets.The Journal of Finance 51(2) (1996): 463-491.

Jones, D.W., Lelby, P.N., Paik, I.K., Oil price shocks and the macroeconomy: What has been learned since 1996?Energy Journal 25 (2004): 1-32.

Katrakilidis, C., Trachnas, E., What drives housing price dynamics in Greece: New evidence from asymmetric ARDL cointegration.Economic Modelling 29 (2012): 1064-1069. 
Kilian, L., Park, C., The impact of oil price shocks on the U.S. stock market. International Economic Review 50(4) (2009): 1267-1287.

Lardic, S., Mignon, V., The impact of oil prices on GDP in European countries: An empirical investigation based on asymmetric cointegration. Energy Policy 34(18) (2006): 3910-3915.

Lardic, S., Mignon, V., Oil prices and economic activity: an asymmetric cointegration approach. Energy Economics 30(3) (2008): 847-855.

Lee, B.J., Yang, C.H., Huang, B.H., Oil price movements and stock market revisited: a case of sector stock price indexes in the G7 countries. Energy Economics 34 (2012): 1284-1300.

Lee, K., Ni, S., On the dynamic effects of oil price shocks: A study using industry level data. Journal of Monetary Economics 49 (2002): 823-852.

Lee,K., Kang, W., Ratti, R.A., Oil price shocks, firm uncertaintyand investment. Macroeconomic Dynamics 15 (2011): 416-436.

Narayan, P.K., Sharma, S.S., New evidence on oil price and firm returns.Journal of Banking and Finance 35(12) (2011): 3253-3262.

Park, J., Ratti, R.A., Oil price shocks and stock markets in the US and 13 European countries.Energy Economics 30 (2008): 2587-2608.

Pesaran, M.H., Shin, Y., 1999. An autoregressive distributed lag modeling approach to cointegration analysis. In: Strom. S. (Ed.). Econometrics and Economic Theory in the 20th Century: The Ragnar Frisch Centennial Symposium.chapter 11. Cambridge University Press. Cambridge.

Pesaran, M.H., Shin, Y., Smith, R.J., Bounds testing approaches to the analysis of level relationships.Journal of Applied Econometrics 16 (2001): 289-326. 
Sadorsky, P., Oil price shocks and stock market activity. Energy Economics 21(5) (1999): 449-469.

Sadorsky, P., Risk factors in stock returns of Canadian oil and gas companies. Energy Economics 23(1) (2001): 17-28.

Serletis, A., Elder, J., Introduction to oil price shocks. Macroeconomic Dynamics 15 (2011): 327-336.

Shin, Y., Yu, B., Greenwood-Nimmo, M., Modelling asymmetric cointegration and dynamic multipliers in a nonlinear ARDL framework.Econometric Methods and Applications.DOI 10 1007/978-1-4899-8008-3_9. (2014): 281-314.

Tsai., C.L., The high-frequency asymmetric response of stock returns to monetary policy for high oil price events. Energy Economics 36 (2013): 166-176.

Williams, B.J., 1938. The Theory of Investment Value.Harvard University Press. Cambridge.

Zhang, D., Oil shock and economic growth in Japan: A nonlinear approach. Energy Economics 30(5) (2008): 2374-2390. 